Swarthmore College

Works

$11-15-2019$

\title{
Realistic Sensitivity Curves For Pulsar Timing Arrays
}

\author{
J. S. Hazboun \\ J. D. Romano
}

Tristan L. Smith

Swarthmore College, tsmith2@swarthmore.edu

Follow this and additional works at: https://works.swarthmore.edu/fac-physics

Part of the Physics Commons

Let us know how access to these works benefits you

\section{Recommended Citation}

J. S. Hazboun, J. D. Romano, and Tristan L. Smith. (2019). "Realistic Sensitivity Curves For Pulsar Timing Arrays". Physical Review D. Volume 100, Issue 10. DOI: 10.1103/PhysRevD.100.104028

https://works.swarthmore.edu/fac-physics/376

This work is brought to you for free by Swarthmore College Libraries' Works. It has been accepted for inclusion in Physics \& Astronomy Faculty Works by an authorized administrator of Works. For more information, please contact myworks@swarthmore.edu. 


\title{
Realistic sensitivity curves for pulsar timing arrays
}

\author{
Jeffrey S. Hazboun $\odot,{ }^{1, *}$ Joseph D. Romano, ${ }^{2}$ and Tristan L. Smith ${ }^{3}$ \\ ${ }^{1}$ University of Washington Bothell, 18115 Campus Way NE, Bothell, Washington 98011, USA \\ ${ }^{2}$ Department of Physics and Astronomy, Texas Tech University, Lubbock, Texas 79409-1051, USA \\ ${ }^{3}$ Department of Physics and Astronomy, Swarthmore College, \\ 500 College Avenue, Swarthmore, Pennsylvania 19081, USA
}

(Received 16 July 2019; published 14 November 2019)

\begin{abstract}
We construct realistic sensitivity curves for pulsar timing array searches for gravitational waves, incorporating both red and white noise contributions to individual pulsar noise spectra, and the effect of fitting to a pulsar timing model. We demonstrate the method on both simulated pulsars and a realistic array consisting of a subset of NANOGrav pulsars used in recent analyses. A comparison between the results presented here and measured upper limit curves from actual analyses shows agreement to tens of percent. The resulting sensitivity curves can be used to assess the detectability of predicted gravitational-wave signals in the nanohertz frequency band in a coherent, flexible, and computationally efficient manner.
\end{abstract}

DOI: $10.1103 /$ PhysRevD.100.104028

\section{MOTIVATION}

Pulsar timing arrays (PTAs) are poised to make the first detection of nanohertz gravitational waves (GWs) in the next 2-5 yrs [1-4]. These galactic-scale GW detectors search for correlations in the times of arrival (TOAs) of the pulses from millisecond pulsars as a signature for the presence of GWs [5-7]. The recent inception of GW astronomy by the advanced LIGO and VIRGO groundbased detectors $[8,9]$ and the multimessenger observations of binary neutron stars [10] have drastically changed our understanding of stellar-mass compact objects. PTAs are poised to complement these observations by observing GWs from binary systems comprised of supermassive black holes (SMBHs) in the centers of distant galaxies.

A common tool used to assess the observability of GW sources across the spectrum are detection sensitivity curves (see, e.g., [11,12] and Fig. 1). These curves are basic "figures of merit," constructed by the developers of GW observatories to assess the sensitivity of current detectors and to predict the sensitivity of future, next-generation detectors. The wider astrophysics community uses detection sensitivity curves as an initial estimate of the ability of a given detector to observe GWs from a particular source.

While detailed sensitivity curves for extant detectors are usually published for each observation run, those for PTAs are often simplified $[11,13,14]$, only including identical white-noise components and often assuming that all pulsar observation epochs are evenly spaced and have the same baseline of observations. When drawn, these curves are often cutoff at the time span of the observations and do not

*hazboun@uw.edu include important insensitivities at frequencies of $1 / \mathrm{yr}$ and $2 / \mathrm{yr}$, due to the fitting for a pulsar's astrometric parameters (Fig. 1). Sensitivity curves should be contrasted with both measured upper limit curves from actual analyses and similar upper limit or detection curves produced by analyzing mock data containing simulated injected signals. A measured upper limit curve is a frequency-dependent illustration of the current limits on the amplitude of a GW signal set by a particular detector (or detectors) analyzing a particular set of real data. Upper limit or detection curves produced by analyzing simulated data typically use the same data analysis routines that are run on the real data and hence are often as computationally involved as the real

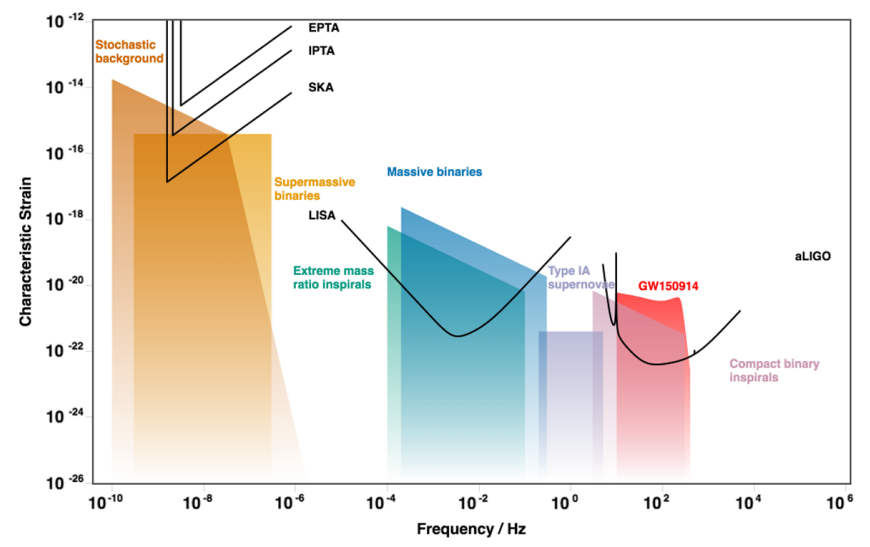

FIG. 1. Sensitivity curves for different GW observations and the predicted spectra of various GW sources. Note, in particular, the (over) simplicity of the PTA sensitivity curves relative to those for LISA and LIGO. The goal of our paper is to construct more realistic PTA sensitivity curves. (Figure produced by gwplotter and based on [11].) 
analyses. Sensitivity curves, on the other hand, are not calculated by analyzing real or simulated data. Rather they simply use properties of the detector network (noise characteristics, configuration, observing schedule, etc.) and expected properties of the signal being searched for to assess the ability of a PTA, for example, to detect such a signal. Sensitivity curves are not meant as a replacement for a full analysis of real PTA data, but instead allow researchers a coherent, flexible, and computationally efficient framework to asses various PTA configurations.

It has long been known that the fit to a pulsar's timing model acts as a filter function $[15,16]$, absorbing frequencies in the pulsar timing data in a predictable manner. These effects have been studied in the context of searches for GWs [3,16,17]. Reference [18] goes one step further, showing how one can mitigate for losses in sensitivity using very-long-baseline interferometry to localize pulsars without explicitly fitting for their positions using the timing data.

Modern PTA data analysis strategies and algorithms are designed with this complication of the timing model fit in mind [19-25]. This formalism was used e.g., in [26] to study PTA sensitivity curves for deterministic and stochastic sources of GWs, calculating sensitivity curves both analytically and numerically, using frequentist and Bayesian methods. The approach in [26] is similar in spirit to ours in that they start from the same likelihood function as we do (Sec. II B), and they use properties of the expected signal-to-noise ratios for deterministic and stochastic GW signals to start to incorporate the effect of timing model fits. Our analysis differs from theirs in that we explicitly identify a component of the likelihood function that encodes both the noise power spectral density in a given pulsar's data set and the effects of the timing model fit. This information is combined with known sources of realistic noise in pulsar timing data, including time-correlated (red) noise, to construct sensitivity curves for individual pulsars. (Reference [27] also discusses the effect of red noise on the sensitivity of pulsar timing searches for GWs, using a Fisher matrix calculation to estimate the errors.) For an array of pulsars, we use the expected signal-to-noise ratio of detection statistics for both deterministic and stochastic GW signals to construct effective sensitivity curves for the whole array.

\section{A. Plan of paper}

In Sec. II, we describe the basic formalism underlying pulsar timing analyses-i.e., timing residuals, timing models, and the effect of fitting to a timing model. This leads us to timing-model-marginalized residuals and their associated transmission functions, which play a key role in the subsequent construction of detection sensitivity curves. In Sec. III, we describe in detail the response of pulsar timing measurements to both deterministic and stochastic GWs. Then, in Sec. IV, we introduce detection statistics for both types of signals. The expressions for their corresponding expected signal-to-noise ratios allow us to read off an effective strain-noise power spectral density for the PTA, which has the interpretation of a detection sensitivity curve. As an application of our analysis, we construct sensitivity curves for the NANOGrav 11-yr pulsars using realistic noise properties and timing model fits, and compare our predicted sensitivities to published upper limits. We conclude in Sec. V. We also include the Appendix, in which we cast the results of an early seminal paper [16] into the more modern notation used in recent pulsar timing analyses.

The calculations provided in this work are packaged in the Python package hasasia, available on the Python Package Index (PyPI) and GitHub, with documentation and tutorials available at ReadTheDocs.

\section{PULSAR TIMING ANALYSES}

Here we review the formalism underlying pulsar timing analyses used in GW searches. Readers interested in more details should see $[16,19,22,23,28]$. The new part of our analysis is the identification of the inverse-noise-weighted transmission function $\mathcal{N}^{-1}(f)$ given in (20), which incorporates both the noise characteristics of the pulsar and the effect of fitting to a timing model; see Sec. II D.

\section{A. Times of arrival and timing residuals}

Let us start with a single pulsar. The measured pulse times of arrival (TOAs) consist of three parts, ${ }^{1}$

$$
t=t^{\operatorname{det}}(\xi)+n+h .
$$

The first term gives the expected TOAs due to deterministic processes, which depend on intrinsic properties of the pulsar (e.g., its spin period, period derivative, ...), extrinsic properties of the pulsar (e.g., its sky location, proper motion, distance from the solar system barycenter, ...), and processes affecting the pulse propagation (e.g., disperion delays due to the interstellar medium, relativistic corrections, ...). The timing model parameters are denoted by $\xi$. The second term is (stochastic) noise intrinsic to the pulsar or to the measurement process itself. The third term is a perturbation to the pulse arrival times induced by $\mathrm{GWs}$, which in general will have contributions from both deterministic and stochastic sources, $h=h^{\mathrm{det}}+h^{\text {stoch }}$.

Timing residuals are then defined by subtracting the expected TOAs (predicted by the timing model for an initial estimate of the model parameters $\xi_{0}$ ) from the measured TOAs,

\footnotetext{
${ }^{1}$ To simplify the notation, we have not included indices to label the particular pulsar $\left(I=1,2, \ldots, N_{\mathrm{p}}\right)$, the individual TOAs $(i=1,2, \ldots, N)$, or the timing model parameters $\left(a=1,2, \ldots, N_{\mathrm{par}}\right)$. If one wants to include those indices explicitly, one should write $t_{I i}=t_{I i}^{\text {det }}\left(\xi_{a}\right)+n_{I i}+h_{I i}$.
} 


$$
\delta t \equiv t-t^{\operatorname{det}}\left(\xi_{0}\right)=M \delta \xi+n+h,
$$

where

$$
\left.M \equiv\left(\frac{\partial t^{\mathrm{det}}}{\partial \xi}\right)\right|_{\xi=\xi_{0}}
$$

is the design matrix. The above expression for $\delta t$ is obtained by Taylor expanding the timing model $t^{\operatorname{det}}(\xi)$ around the initial parameter estimates $\xi_{0}$, assuming that the initial estimates are close enough to the true values that only first-order terms in the parameter deviations $\delta \xi$ are needed in the expansion. The design matrix $M$ is a rectangular matrix of dimension $N \times N_{\text {par }}$, with components $M_{i a}$. Each column of the design matrix encodes the linearized fit to one parameter in the timing model.

\section{B. Fitting to a timing model}

From the form of (2), one sees that errors $\delta \xi$ in our original estimate $\xi_{0}$ of the timing model parameters lead to deterministic features in the timing residuals. For example, an error in the pulse period leads to timing residuals that grow linearly with time, $\delta t \sim t$, while an error in the period derivative leads to residuals that grow quadratically with time, $\delta t \sim t^{2}$. Thus, we can improve our estimates of the timing model parameters by fitting for $\delta \xi$ in our linear timing model for the residuals.

This can be done in two ways, both of which take the likelihood function

$$
\begin{aligned}
& p\left(\delta t \mid \delta \xi, C_{n}, C_{h}, \theta\right) \\
& \quad \propto \exp \left[-\frac{1}{2}(\delta t-M \delta \xi-h(\theta))^{T} C^{-1}(\delta t-M \delta \xi-h(\theta))\right]
\end{aligned}
$$

as the starting point. In the above expression,

$$
C \equiv C_{n}+C_{h}
$$

is the noise covariance matrix, which has contributions from both detector noise $C_{n}$ (i.e., noise intrinsic to the pulsar and from the measurement process) and a potential GW background $C_{h}$. The term $h(\theta)$ are the timing residuals induced by a deterministic GW source (e.g., the expected waveform from an individual SMBH binary parametrized by $\theta$ ).

(i) The first approach to fitting to the timing model is to maximize the likelihood function with respect to the parameter deviations $\delta \xi$. Since $\delta \xi$ appears linearly in the expression for the timing residuals (quadratically in the argument of the exponential), the maximization is easy to do. One obtains the standard result,

$$
\delta \xi_{\mathrm{ML}}=\left(M^{T} C^{-1} M\right)^{-1} M^{T} C^{-1} \delta t .
$$

From these maximum-likelihood estimates, we can then form postfit residuals,

$$
\begin{gathered}
\delta t^{\mathrm{post}} \equiv \delta t-M \delta \xi_{\mathrm{ML}}=R \delta t, \\
R \equiv 1-M\left(M^{T} C^{-1} M\right)^{-1} M^{T} C^{-1} .
\end{gathered}
$$

Note that $R$ is an $N \times N$ matrix that implements the fit to the linear timing model; it depends in general on both the timing model (via $M$ ) and the detector noise (via $C$ ). One can show that $R$ is a projection operator $\left(R^{2}=R\right)$, and hence not invertible.

(ii) The second approach to fitting to the timing model is to marginalize the likelihood function over the parameter deviations $\delta \xi$, assuming flat priors for $\delta \xi$. The result of this marginalization is the timing-model-marginalized (TMM) likelihood function [20,22],

$$
\begin{aligned}
& p\left(\delta t \mid C_{n}, C_{h}, \theta\right) \\
& \quad \propto \exp \left[-\frac{1}{2}(\delta t-h(\theta))^{T} G\left(G^{T} C G\right)^{-1} G^{T}(\delta t-h(\theta))\right],
\end{aligned}
$$

where $G$ is an $N \times\left(N-N_{\text {par }}\right)$ matrix constructed from a singular-value decomposition of the design matrix

$$
M=U S V^{T}, \quad U=(F, G) .
$$

Here $U$ and $V^{T}$ are orthogonal matrices of dimensions $N \times N$ and $N_{\text {par }} \times N_{\text {par }}$, respectively, and $\mathrm{S}$ is an $N \times N_{\text {par }}$ diagonal matrix with the singular values along the diagonal. $F$ is the $N \times N_{\text {par }}$ matrix containing the first $N_{\text {par }}$ columns of $U$ and $G$ is the $N \times\left(N-N_{\text {par }}\right)$ matrix consisting of the other columns of $U$. Note that $G$ depends only on the timing model (via $M$ ) and not on the noise. In terms of components, $G \equiv G_{i \alpha}$, where $\alpha=1,2, \ldots, N-N_{\text {par }}$. Using $G$, one can construct associated TMM residuals,

$$
r \equiv G^{T} \delta t
$$

which are orthogonal to the timing model. Since $U$ is a unitary matrix, it follows that $\left[G^{T} G\right]_{\alpha \beta}=\delta_{\alpha \beta}$. For white noise (i.e., $C$ proportional to the identity matrix), we have the identity $R=G G^{T}$.

Although both approaches for fitting to the timing model have been used in the past (compare for instance [28] with [23]), in this paper we will use the second approach, given that it is the one used most often for current pulsar timing array searches for GWs.

\section{Transmission functions}

The process of fitting to a timing model removes power from the postfit or TMM residuals. This can be easily 


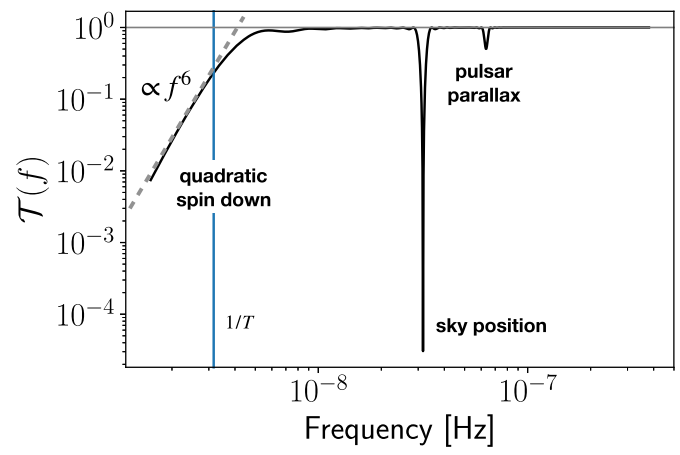

(a)

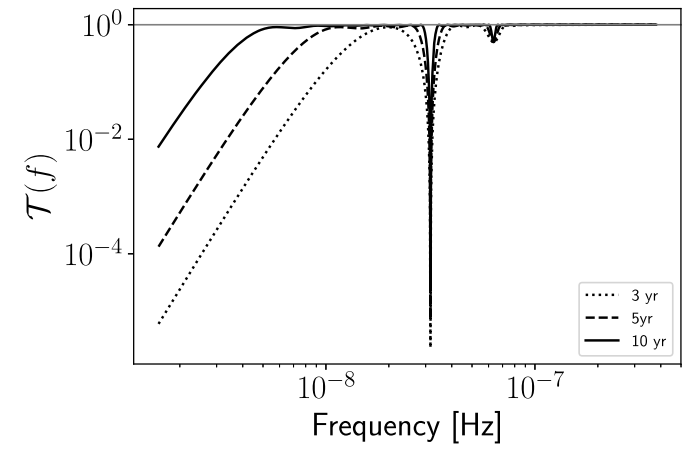

(b)

FIG. 2. Transmission functions corresponding to a fit to a simple timing model. Panel (a): The $\sim f^{6}$ dependence of power absorption at low frequencies is due to fitting the quadratic spin-down model for the pulsar; see [16]. Other absorption dips, due to fits for the sky position and distance to the pulsar (parallax), can also be seen. The blue vertical line corresponds to a frequency of $1 / T$, where $T$ is the observation time. Panel (b): Dependence of the transmission function on the duration of the observation. The spikes become deeper and narrower, and the knee frequency shifts to the left, as the observing time $T$ increases.

demonstrated by calculating the variance of the TMM residuals $r \equiv G^{T} \delta t$. One finds

$$
\sigma_{r}^{2}=\int_{0}^{\infty} \mathrm{d} f \mathcal{T}(f) P(f)
$$

where $P(f)$ is the (one-sided) power spectral density of the original (pre-fit) timing residuals $\delta t$, and

$$
\mathcal{T}(f) \equiv \frac{1}{N} \sum_{k, l}\left(G G^{T}\right)_{k l} e^{i 2 \pi f\left(t_{k}-t_{l}\right)} .
$$

Here $t_{k}$ and $t_{l}$ denote the times of arrival of the $k$ th and $l$ th pulses, with $k, l=1,2, \ldots, N$. The function $\mathcal{T}(f)$ has the interpretation of a transmission function, selectively removing power associated with the timing model fit. A plot of $\mathcal{T}(f)$ for a simple timing model consisting of quadratic spin-down (i.e., fitting to the phase offset, spin period, and period derivative of the pulsar), the pulsar's sky position, and the distance to the pulsar is shown in Fig. 2(a). Note that fitting to the sky position absorbs power at and around a frequency of 1 /year, corresponding to the Earth's yearly orbital motion around the Sun. Fitting to the pulsar distance absorbs power at a frequency of $2 / \mathrm{yr}$, which corresponds to a parallax measurement. The quadratic spin-down parameter fit acts as a high-pass filter, absorbing frequencies substantially below $1 / T$, where $T$ is the time span of the data. The effect of the observing time on the shape of the transmission function is shown in Fig. 2(b).

Pulsars in binaries famously have additional components to the timing model that take into account the various Doppler shifts due to binary motion and relativistic effects, if the line-of-sight passes by the companion (Shapiro delay) or if the binary is in a tight enough orbit to observe the loss of power due to GWs [29]. These components of the timing model have a minimal effect on sensitivity curves for GWs as the frequencies in question are much higher than those of the sources for which PTAs are searching. We do not include these components when simulating pulsar design matrices, but we will see the (mostly subtle) changes they make when looking at the design matrices of real pulsar data.

Finally, we note that one can also calculate an analogous transmission function associated with the postfit timing residuals $\delta t^{\text {post }} \equiv R \delta t$. One finds

$$
\sigma_{\text {post }}^{2}=\int_{0}^{\infty} \mathrm{d} f \mathcal{T}_{R}(f) P(f)
$$

where

$$
\mathcal{T}_{R}(f) \equiv \frac{1}{N} \sum_{k, l} R_{k l} e^{i 2 \pi f\left(t_{k}-t_{l}\right)}
$$

This $R$-matrix transmission function was originally described in [16], although from a slightly different perspective. In the Appendix, we cast the approach of [16] into the more modern $R$-matrix notation.

\section{Inverse-noise-weighted transmission function}

It turns out that there is another way of obtaining a quantity that behaves like a transmission function by working directly with the TMM likelihood (9). The argument of the exponential can be written as $-\frac{1}{2} \chi^{2}$, where

$$
\chi^{2} \equiv(\delta t-h(\theta))^{T} G\left(G^{T} C G\right)^{-1} G^{T}(\delta t-h(\theta)) .
$$

If we write this in the Fourier domain by substituting

$$
h_{k}(\theta) \equiv h\left(t_{k} ; \theta\right)=\int_{-f_{\mathrm{Nyq}}}^{f_{\mathrm{Nyq}}} \mathrm{d} f \tilde{h}(f ; \theta) e^{i 2 \pi f t_{k}},
$$




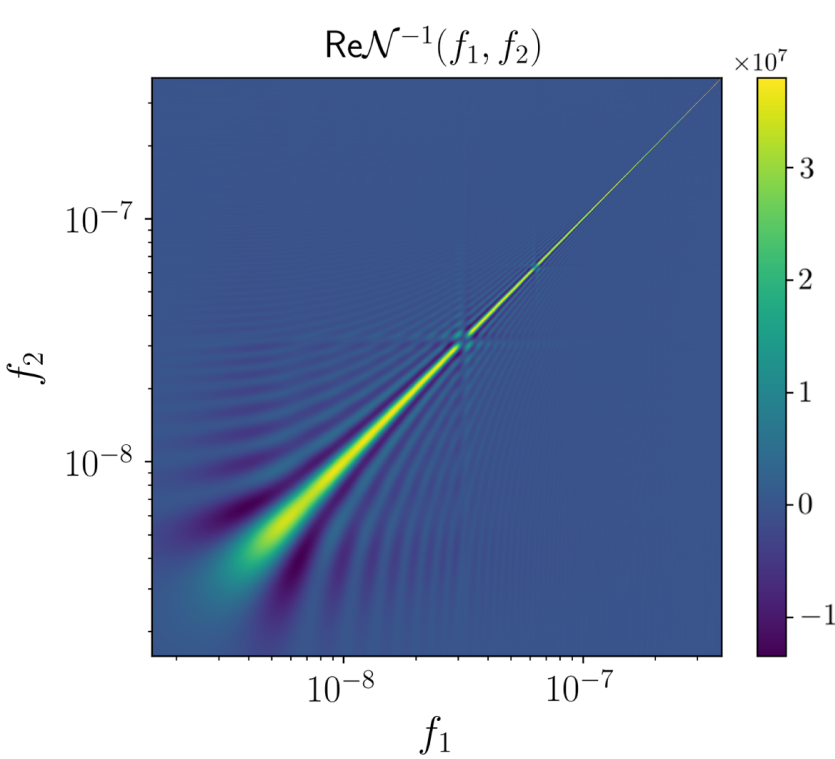

(a)

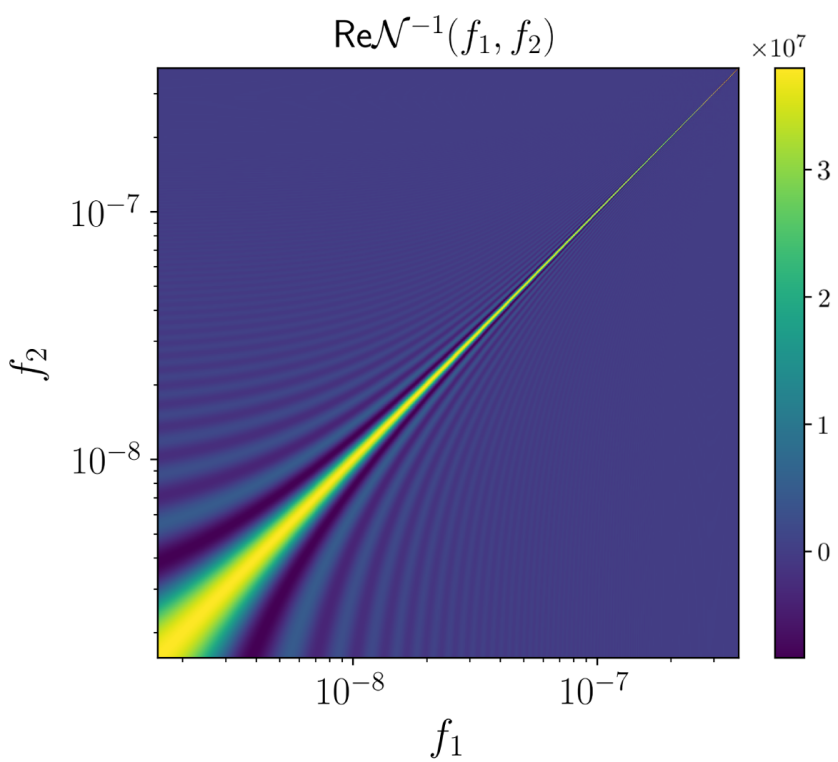

(b)

FIG. 3. Two-dimensional plot of the real part of the function $\mathcal{N}^{-1}\left(f_{1}, f_{2}\right)$ for $f_{1}, f_{2}>0$ plotted on log-scale axes. Panel (a): $\operatorname{Re}\left[\mathcal{N}^{-1}\left(f_{1}, f_{2}\right)\right]$ for white noise ( $C$ is proportional to the identity matrix) and a fit to the simple quadratic spin-down timing model described in the main text. The small amplitude in the bottom-left hand corner of the plot is due to the absorption of power by the timing model fit at and below $1 / T$. There is also suppression at $f_{1}=f_{2}=1 / \mathrm{yr}$ and $f_{1}=f_{2}=2 / \mathrm{yr}$. Panel (b): For comparison, a twodimensional plot of $\operatorname{Re}\left[\mathcal{N}^{-1}\left(f_{1}, f_{2}\right)\right]$ for white noise, but without performing a timing model fit (so $G$ is proportional to the identity matrix).

where $t_{k} \equiv k \Delta t$ and $f_{\mathrm{Nyq}} \equiv 1 /(2 \Delta t)$, we find

$$
\begin{aligned}
\chi^{2}= & 2 T \int_{-f_{\mathrm{Nyq}}}^{f_{\mathrm{Nyq}}} \mathrm{d} f \int_{-f_{\mathrm{Nyq}}}^{f_{\mathrm{Nyq}}} \mathrm{d} f^{\prime}(\widetilde{\delta} t(f)-\tilde{h}(f ; \theta)) \\
& \times \mathcal{N}^{-1}\left(f, f^{\prime}\right)\left(\widetilde{\delta t^{*}}\left(f^{\prime}\right)-\tilde{h}^{*}\left(f^{\prime} ; \theta\right)\right),
\end{aligned}
$$

where

$\mathcal{N}^{-1}\left(f, f^{\prime}\right) \equiv \frac{1}{2 T} \sum_{k, l} e^{i 2 \pi f t_{k}}\left[G\left(G^{T} C G\right)^{-1} G^{T}\right]_{k l} e^{-i 2 \pi f^{\prime} t_{l}}$

The quantity $\mathcal{N}^{-1}\left(f, f^{\prime}\right)$ is a function of two frequencies, $\left(f, f^{\prime}\right)$, but it turns out to be diagonally dominated, with the majority of its support on the diagonal $f=f^{\prime}$, as shown in Fig. 3(a). (The broadening of the diagonal band at low frequencies is an artifact of using log-scale axes for the frequencies.) The diagonal component,

$$
\mathcal{N}^{-1}(f) \equiv \frac{1}{2 T} \sum_{k, l}\left[G\left(G^{T} C G\right)^{-1} G^{T}\right]_{k l} e^{i 2 \pi f\left(t_{k}-t_{l}\right)},
$$

and three off diagonal cross sections of $\mathcal{N}^{-1}\left(f, f^{\prime}\right)$ are shown in Fig. 4. (The fact that the off diagonal cross sections are curved in panel (a) of Fig. 4 is again due to using log-scale axes for the frequencies.) A few remarks are in order: (i) For this particular example, the diagonal component $\mathcal{N}^{-1}(f)$ is identical in shape with the transmission function $\mathcal{T}(f)$ shown in Fig. 2(a). The amplitude of $\mathcal{N}^{-1}(f)$ differs from $\mathcal{T}(f)$ by a constant factor $1 / P(f)=1 /\left(2 \sigma^{2} \Delta t\right)$, corresponding to a white noise covariance matrix. ${ }^{2}$ Thus, for white noise,

$$
\mathcal{N}^{-1}(f)=\mathcal{T}(f) / P(f) .
$$

This is illustrated in Fig. 5(a). If we also include red noise in the noise covariance matrix $C$ by taking

$$
\begin{gathered}
C_{i j}=\int_{0}^{f_{\mathrm{Nyq}}} \mathrm{d} f \cos \left[2 \pi f\left(t_{i}-t_{j}\right)\right] P(f), \\
P(f)=2 \sigma^{2} \Delta t+A^{2} f^{-\gamma}, \quad \gamma>0,
\end{gathered}
$$

then the relationship between $\mathcal{N}^{-1}(f)$ and $\mathcal{T}(f) / P(f)$ is only approximate,

$$
\mathcal{N}^{-1}(f) \approx \mathcal{T}(f) / P(f) .
$$

This is illustrated in Fig. 5(b).

(ii) Away from the dip at $1 / \mathrm{yr}$, where there is suppression of power due to the timing model fit to the pulsar sky

\footnotetext{
${ }^{2}$ For our white noise simulations, we take $P(f)=2 \sigma^{2} \Delta t$, with $\sigma=100 \mathrm{~ns}$ and $\Delta t=\mathrm{yr} / 20$. These numerical values are often chosen for pulsar timing simulations.
} 


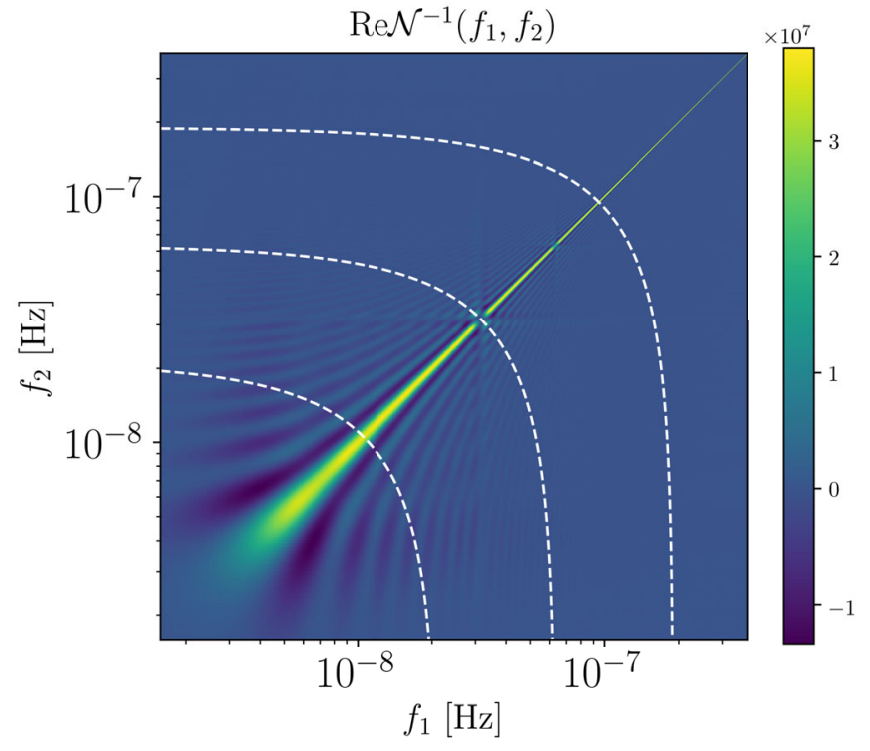

(a)

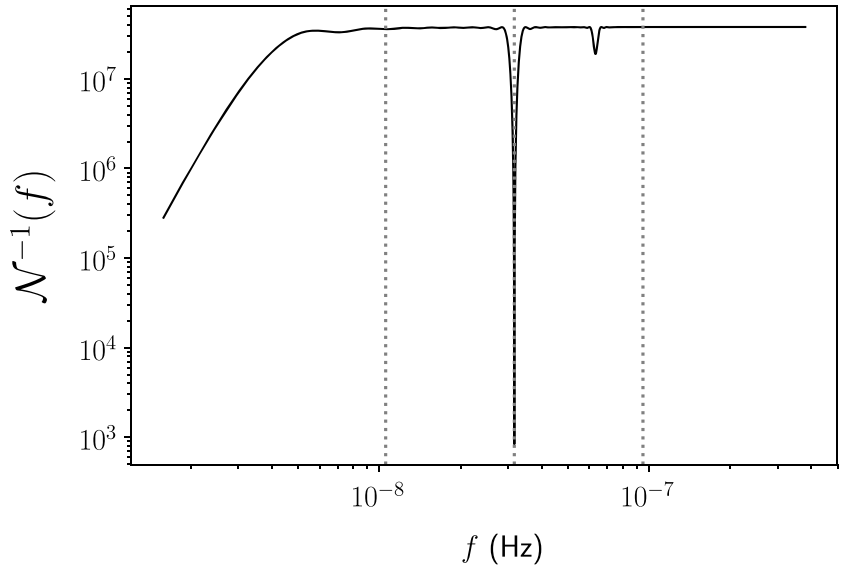

(b)

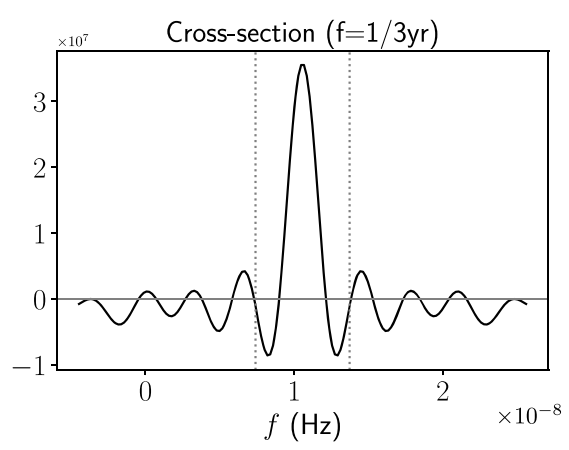

(c)

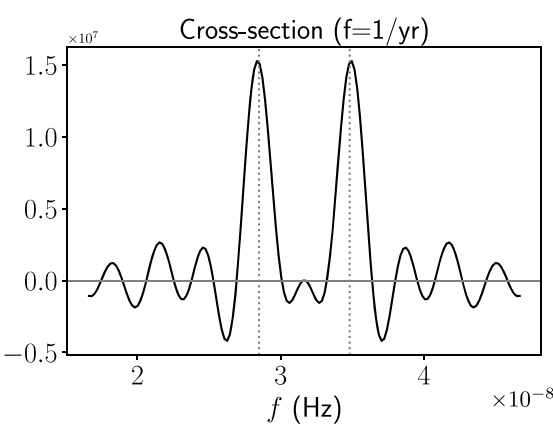

(d)

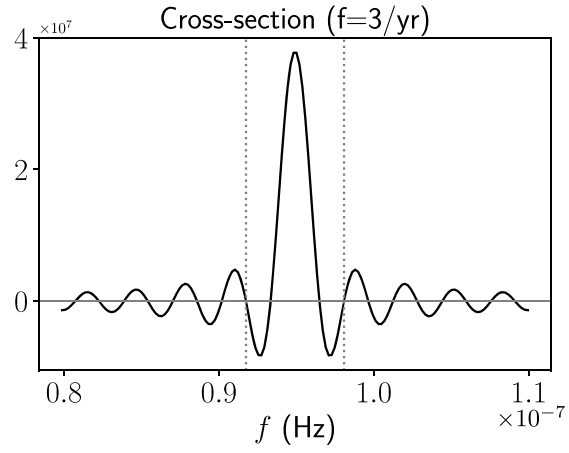

(e)

FIG. 4. Diagonal and off diagonal cross sections of $\mathcal{N}^{-1}\left(f_{1}, f_{2}\right)$. Panel (a): $\operatorname{Re}\left[\mathcal{N}^{-1}\left(f_{1}, f_{2}\right)\right]$ from Fig. 3(a) with off diagonal cross sections shown by white-dotted lines. Panel (b): Diagonal component $\mathcal{N}^{-1}(f)$ (the dotted vertical lines show the frequencies of the off diagonal cross sections). Panels (c)-(e): Real part of the off-diagonal cross sections of $\mathcal{N}^{-1}\left(f_{1}, f_{2}\right)$ for $f=1 /(3 \mathrm{yr}), f=1 / \mathrm{yr}$, and $f=3 /$ yr. Away from $1 /$ yr, the off diagonal cross sections are proportional to Dirichlet sinc functions (the dotted vertical lines indicate offsets of $\pm 1 / T)$.

position, the off diagonal cross sections are proportional to Dirichlet sinc functions,

$$
\mathcal{D}_{N}\left(\left(f-f^{\prime}\right) \Delta t\right) \equiv \frac{1}{N} \frac{\sin \left[N \pi\left(f-f^{\prime}\right) \Delta t\right]}{\sin \left[\pi\left(f-f^{\prime}\right) \Delta t\right]} .
$$

When multiplied by $T$, a Dirichlet sinc function can be thought of as finite-time approximation to the Dirac delta function-i.e., $\quad \delta\left(f-f^{\prime}\right) \simeq T \mathcal{D}_{N}\left((f-f)^{\prime} \Delta t\right)$. Dirichlet sinc functions arise when taking the Fourier transform of a discretely sampled rectangular window of duration $T=N \Delta t$; see e.g., [30]. This diagonally dominated behavior is what you would expect for $\mathcal{N}^{-1}\left(f, f^{\prime}\right)$ if one had only Gaussian-stationary noise. This is the case if one does not have to fit a timing model [Fig. 3(b)]. Then one can simply replace $G$ by the identity matrix, for which

$$
\begin{aligned}
\mathcal{N}^{-1}\left(f, f^{\prime}\right) & =\frac{1}{2 T} \sum_{k, l} e^{i 2 \pi f t_{k}\left[C^{-1}\right]_{k l} e^{-i 2 \pi f^{\prime} t_{l}}} \\
& \simeq P^{-1}(f) \delta_{f f^{\prime}} .
\end{aligned}
$$

The approximate equality in the above equation is a consequence of the Karhunen-Loeve theorem, which states that the discrete Fourier transform operation defined by the unitary matrix $U_{j k} \equiv \frac{1}{N} e^{-i 2 \pi j k / N}$ approximately diagonalizes a stationary covariance matrix in the limit that the observation time $T=N \Delta t$ is much larger than the correlation time of the noise.

(iii) Since fitting to a timing model introduces nonstationarities into the TMM residuals [22], one cannot directly appeal to the Karhunen-Loeve theorem for the general expression (19). One needs to explicitly check the 


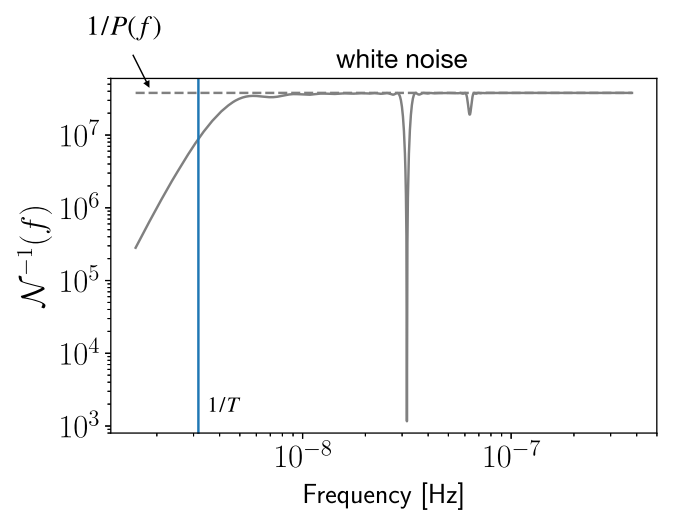

(a)

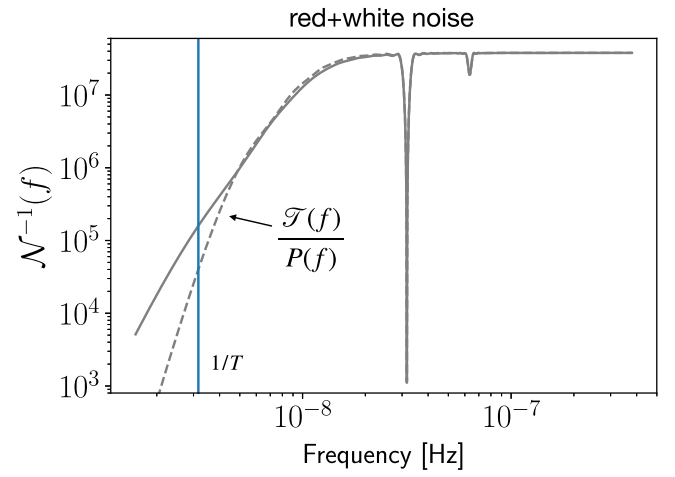

(b)

FIG. 5. Plots of the inverse-noise-weighted transmission function $\mathcal{N}^{-1}(f)$ for the simple quadratic spin-down model described in the main text, and for white noise (a) and red + white noise (b). Panel (a): For white noise, the amplitude of $\mathcal{N}^{-1}(f)$ is set by the constant value of $1 / P(f)$ indicated by the horizontal dashed line. Panel (b): The curved dashed line is a plot of $\mathcal{T}(f) / P(f)$, which is an approximation to $\mathcal{N}^{-1}(f)$ for $P(f)$ consisting of red + white noise.

validity of the diagonal approximation for $\mathcal{N}^{-1}\left(f, f^{\prime}\right)$ as we have done in Figs. 3 and 4. We have also numerically computed the sum of $\mathcal{N}^{-1}\left(f, f^{\prime}\right)$ over the full twodimensional array of frequencies $\left(f, f^{\prime}\right)$ and compared that to the sum of $\mathcal{N}^{-1}\left(f, f^{\prime}\right)$ just along the diagonal $f=f^{\prime}$. Even for the more challenging case of a red + white noise covariance matrix [Fig. 5(b] and a fit to the quadratic spin-down model, the two summations agree to within $\approx 6 \%$.

(iv) Even though we transformed the likelihood function into the Fourier domain to obtain expressions (19) and (20) for $\mathcal{N}^{-1}\left(f, f^{\prime}\right)$ and $\mathcal{N}^{-1}(f)$, those expressions are calculable directly in terms of the times-of-arrival $t_{k}$ and $t_{l}$. This means that these expressions for the inverse-noiseweighted transmission functions are valid even for irregularly sampled data. Our use of a sampling period $\Delta t$ is only for convenience when discussing power spectra or when calculating the Fourier transform of the GW response; it is not a requirement for our sensitivity curve analysis.

\section{TIMING RESIDUAL RESPONSE TO GRAVITATIONAL WAVES}

To proceed further in our calculation of pulsar timing sensitivity curves, we need to describe in more detail the timing residual response of a pulsar to an incident GW. We will consider both deterministic and stochastic sources of GWs. Interested readers should see [19-22] for more details. Readers already familiar with this material can skip to Sec. IV, where we show how the inverse-noise-weighted transmission function $\mathcal{N}^{-1}(f)$ enters into expressions for the expected signal-to-noise ratio of standard statistics used to search for both deterministic and stochastic GW signals.

\section{A. Response to a single deterministic source}

We will start by writing down the metric perturbations $h_{a b}(t, \vec{x})$ for a single deterministic source emitting plane
GWs in the direction $\hat{k}$ (Fig. 6). To do this we introduce two coordinate frames: one associated with the solar system barycenter (SSB) and the other associated with the propagation of the GW. We will assume that the source has a symmetry axis (e.g., the direction of the orbital angular momentum vector $\vec{L}$ for a binary system) and that the symmetry axis makes an angle $l$ with respect to the line of sight $\hat{k}$ from the $\mathrm{GW}$ source to the solar system barycenter, and an angle $\psi$ with respect to the vector $\hat{l}$ when projected onto the plane perpendicular to $\hat{k}$ (Fig. 7).

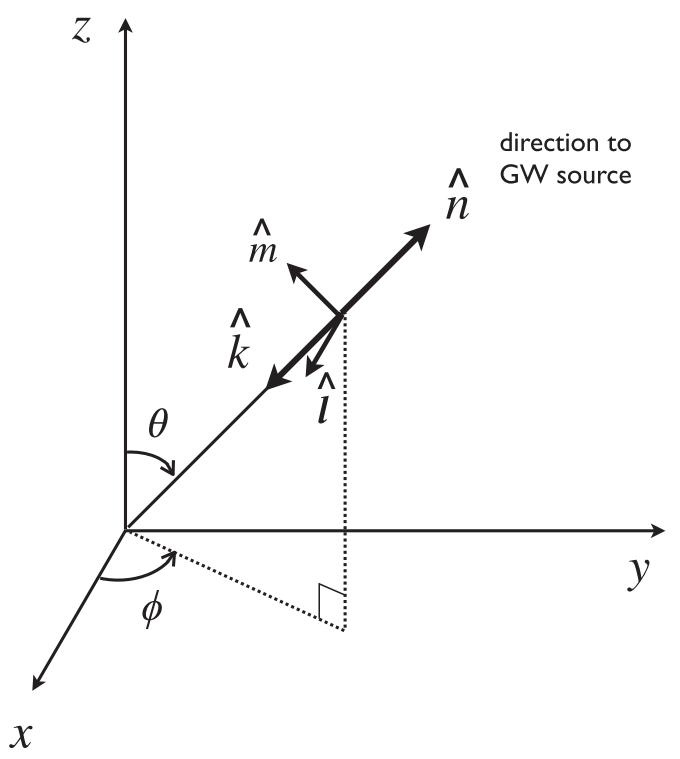

FIG. 6. Definition of the unit vectors $\hat{k}, \hat{l}, \hat{m}$. The direction of propagation of the $\mathrm{GW}, \hat{k}$, is opposite the direction to the source, $\hat{n}$. The unit vectors $\hat{l}, \hat{m}$ are in the plane perpendicular to $\hat{k}$, and point in directions of constant declination and right ascension, respectively. 


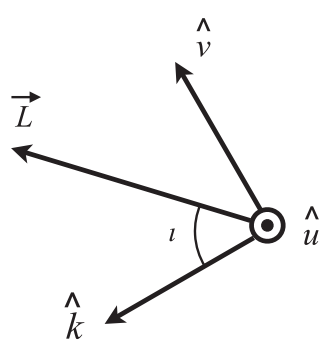

(a)

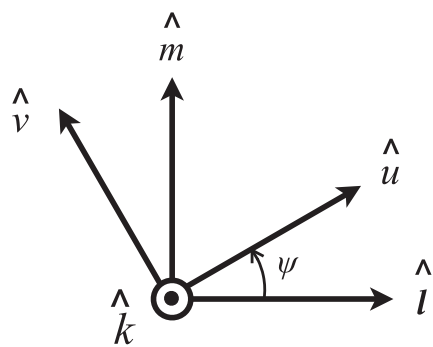

(b)
FIG. 7. Relation between the unit vectors $\hat{l}, \hat{m}$ and $\hat{u}, \hat{v}$. Definition of (a) inclination angle $l$ and (b) polarization angle $\psi$. Here $\vec{L}$ is the angular momentum vector and $\hat{k}$ is the propagation direction of the GW. The vectors $\hat{l}, \hat{m}$ and $\hat{u}, \hat{v}$ are orthogonal unit vectors in the plane perpendicular to $\hat{k}$, defined by (27) and (29), respectively.

The vectors $\hat{k}, \hat{l}, \hat{m}$ are defined in the solar system barycenter frame by

$$
\begin{aligned}
\hat{k} & =(-\sin \theta \cos \phi,-\sin \theta \sin \phi,-\cos \theta) \equiv-\hat{r}, \\
\hat{l} & =(\sin \phi,-\cos \phi, 0) \equiv-\hat{\phi}, \\
\hat{m} & =(-\cos \theta \cos \phi,-\cos \theta \sin \phi, \sin \theta) \equiv-\hat{\theta},
\end{aligned}
$$

where $(\theta, \phi)$ are the standard polar and azimuthal angles on the 2-sphere in equatorial coordinates, and the origin of coordinates is at the solar system barycenter. The right ascension $\alpha$ and declination $\delta$ of a source are given in terms of $\theta$ and $\phi$ by $\alpha=\phi$ and $\delta=\pi / 2-\theta$.

The angles $l$ and $\psi$ are the inclination and polarization angles of the source, respectively. They can be written in terms of the unit vectors $\hat{k}, \hat{l}, \hat{L} \equiv \vec{L} /|\vec{L}|$, and $\hat{u}$ via

$$
\cos l \equiv \hat{k} \cdot \hat{L}, \quad \cos \psi \equiv \hat{u} \cdot \hat{l},
$$

where

$$
\hat{u} \equiv \frac{\hat{L} \times \hat{k}}{|\hat{L} \times \hat{k}|}, \quad \hat{v} \equiv \hat{k} \times \hat{u}
$$

are two orthogonal unit vectors in the plane perpendicular to $\hat{k}$ (Fig. 7). Note that $l=0$ or $\pi$ corresponds to the orbital plane being seen face-on or face-off; $l=\pi / 2$ or $3 \pi / 2$ corresponds to seing the orbital plane edge on. The unit vectors $\hat{u}, \hat{v}$ are related to $\hat{l}, \hat{m}$ by a rotation around $\hat{k}$ through the angle $\psi$ as shown in Fig. 7(b).

From $\hat{u}$ and $\hat{v}$, we can construct a preferred set of polarization tensors,

$$
\begin{aligned}
& \epsilon_{a b}^{+}(\hat{k}, \psi) \equiv \hat{u}_{a} \hat{u}_{b}-\hat{v}_{a} \hat{v}_{b}, \\
& \epsilon_{a b}^{\times}(\hat{k}, \psi) \equiv \hat{u}_{a} \hat{v}_{b}+\hat{v}_{a} \hat{u}_{b},
\end{aligned}
$$

where $a, b$ in the above and following expressions ${ }^{3}$ refer to spatial indices in transverse-traceless coordinates $(t, \vec{x}) \equiv$ $\left(t, x^{a}\right)$, with $a=1,2,3$. Using these polarization tensors, we can expand the metric perturbations,

$$
\begin{aligned}
h_{a b}(t, \vec{x})= & h_{+}(t-\hat{k} \cdot \vec{x} / c ; \imath) \epsilon_{a b}^{+}(\hat{k}, \psi) \\
& +h_{\times}(t-\hat{k} \cdot \vec{x} / c ; \iota) \epsilon_{a b}^{\times}(\hat{k}, \psi)
\end{aligned}
$$

or, equivalently,

$$
\begin{aligned}
h_{a b}(t, \vec{x})= & \int_{-\infty}^{\infty} \mathrm{d} f\left[\tilde{h}_{+}(f ; \imath) \epsilon_{a b}^{+}(\hat{k}, \psi)+\tilde{h}_{\times}(f ; l) \epsilon_{a b}^{\times}(\hat{k}, \psi)\right] \\
& \times e^{i 2 \pi f(t-\hat{k} \cdot \vec{x} / c)},
\end{aligned}
$$

where $\tilde{h}_{+, \times}(f ; l)$ are the Fourier transforms of $h_{+, \times}(t ; \imath)$. The timing residual response of a pulsar to such a deterministic GW is then [31],

$$
h(t ; \hat{k}, l, \psi)=\int_{-\infty}^{\infty} \mathrm{d} f \tilde{h}(f ; \hat{k}, l, \psi) e^{i 2 \pi f t},
$$

where

$\tilde{h}(f ; \hat{k}, \imath, \psi)=R^{+}(f, \hat{k}, \psi) \tilde{h}_{+}(f ; \iota)+R^{\times}(f, \hat{k}, \psi) \tilde{h}_{\times}(f ; \iota)$,

with

$$
\begin{aligned}
R^{+, \times}(f, \hat{k}, \psi) \equiv & \frac{1}{i 2 \pi f} \frac{1}{2} \frac{\hat{p}^{a} \hat{p}^{b}}{1+\hat{p} \cdot \hat{k}} \epsilon_{a b}^{+, \times}(\hat{k}, \psi) \\
& \times\left(1-e^{-i 2 \pi f D(1+\hat{k} \cdot \hat{p}) / c}\right) .
\end{aligned}
$$

Here $\hat{p}^{a}$ is a unit vector pointing from the solar system barycenter to the pulsar, and $D$ is the distance to the pulsar. The function $R^{+, \times}(f, \hat{k}, \psi)$ is the timing residual response function of a pulsar to a monochromatic plane GW propagating in direction $\hat{k}$, with frequency $f$, polarization ,$+ \times$, and polarization angle $\psi$. The two terms in the response function are called the "Earth term" and "pulsar term", respectively, since they involve sampling the GW phase at Earth and at the location of the pulsar, a distance $D$ away from Earth. The factor of $1 /(i 2 \pi f)$ comes from the fact that we are working with timing residuals, as opposed to Doppler shifts in the pulse frequency.

For the analyses that we will do in this paper, we will typically ignore the pulsar-term contribution to the timing residual response to GWs, as this term will not contribute to the cross-power when correlating the signal associated with

\footnotetext{
${ }^{3}$ In footnote 1, we mentioned that $a, b, \ldots$ would also be used to label the individual timing parameters $\xi_{a}$, with $a=1,2, \ldots, N_{\text {par }}$. It should be clear from the context whether an $a$ index refers to spatial indices (as above) or timing model parameters.
} 
distinct pulsars. [The separation between pulsars $(\sim \mathrm{kpc})$ is much greater the wavelengths of the GWs that we are sensitive to, which are of order $\lesssim 10$ lyr.] There is a contribution, however, to the autocorrelated power for a single pulsar, which comes from the exponential part of $\left|R^{+, \times}(f, \hat{k}, \psi)\right|^{2}$,

$$
\begin{aligned}
\mid 1 & -\left.e^{-i 2 \pi f D(1+\hat{k} \cdot \hat{p}) / c}\right|^{2} \\
\quad & =2[1-\cos (2 \pi f D(1+\hat{k} \cdot \hat{p}) / c)] \simeq 2,
\end{aligned}
$$

where we have ignored the cosine term since it is a rapidly oscillating function of the GW propagation direction $\hat{k}$ and hence does not contribute significantly when summed over the sky. The value "2" corresponds to the sum of the EarthEarth and pulsar-pulsar autocorrelation terms.

\section{Circular binaries}

To proceed further, we need to specify the form of $h_{+, \times}(t ; l)$ or its Fourier transform $\tilde{h}_{+, \times}(f ; \imath)$. For example, for a circular binary,

$$
\begin{aligned}
& h_{+}(t ; l)=h_{0}(t)\left(\frac{1+\cos ^{2} \imath}{2}\right) \cos 2 \Phi(t), \\
& h_{\times}(t ; l)=h_{0}(t) \cos \imath \sin 2 \Phi(t)
\end{aligned}
$$

where $\Phi(t)$ is the orbital phase and $h_{0}(t)$ is a dimensionless amplitude given by

$$
h_{0}(t)=\frac{4 c}{D_{L}}\left(\frac{G \mathcal{M}_{\mathrm{c}}}{c^{3}}\right)^{5 / 3} \omega(t)^{2 / 3} .
$$

Here $D_{L}$ is the luminosity distance to the source, $\mathcal{M}_{\mathrm{c}} \equiv$ $\left(m_{1} m_{2}\right)^{3 / 5} /\left(m_{1}+m_{2}\right)^{1 / 5}$ is the chirp mass of the binary system, and $\omega(t)$ is the instantaneous orbital angular frequency, $\Phi(t)=\int^{t} \mathrm{~d} t^{\prime} \omega\left(t^{\prime}\right)$. For an evolving binary system,

$$
\frac{\mathrm{d} \omega}{\mathrm{d} t}=\frac{96}{5}\left(\frac{G \mathcal{M}_{c}}{c^{3}}\right)^{5 / 3} \omega(t)^{11 / 3}
$$

which is a consequence of energy balance between the radiated power in GWs and the orbital energy lost by the binary system. The instantaneous GW frequency $f(t)$ is related to the orbital frequency $\omega(t)$ via $\omega(t)=\pi f(t)$.

It is fairly easy to show that for a binary system consisting of SMBHs with component masses $M \sim 10^{9} M_{\text {solar }}$ in the PTA sensitivity band (nHz), the frequency evolution of the binary over a decade-long observation is roughly 4 orders of magnitude smaller than the frequency bin width, $1 / T$, set by the total observation time $T \sim 10 \mathrm{yr}$. Thus, for the purposes of this paper, we will take our deterministic source to be a monochromatic binary with $f(\tau)=f_{0}=$ const.
With this simplification, Eqs. (37) and (38) become

$$
\begin{aligned}
& h_{+}\left(t ; l, \phi_{0}\right)=h_{0}\left(\frac{1+\cos ^{2} \iota}{2}\right) \cos \left(2 \pi f_{0} t+\phi_{0}\right), \\
& h_{\times}\left(t ; l, \phi_{0}\right)=h_{0} \cos \imath \sin \left(2 \pi f_{0} t+\phi_{0}\right),
\end{aligned}
$$

where $\phi_{0}$ is the initial phase and $h_{0}$ is the (constant) strain amplitude,

$$
h_{0}=\frac{4 c}{D_{L}}\left(\frac{G \mathcal{M}_{\mathrm{c}}}{c^{3}}\right)^{5 / 3}\left(\pi f_{0}\right)^{2 / 3}
$$

The Fourier transforms of $h_{+, \times}\left(t ; l, \phi_{0}\right)$ are then

$$
\begin{aligned}
\tilde{h}_{+}\left(f ; l, \phi_{0}\right)= & h_{0}\left(\frac{1+\cos ^{2} \imath}{2}\right) \\
& \times \frac{1}{2}\left[e^{i \phi_{0}} \delta\left(f-f_{0}\right)+e^{-i \phi_{0}} \delta\left(f+f_{0}\right)\right], \\
\tilde{h}_{\times}\left(f ; l, \phi_{0}\right)= & h_{0} \cos \imath \\
& \times \frac{1}{2 i}\left[e^{i \phi_{0}} \delta\left(f-f_{0}\right)-e^{-i \phi_{0}} \delta\left(f+f_{0}\right)\right] .
\end{aligned}
$$

But since the signals are observed for only a finite duration, the Dirac delta functions $\delta\left(f \mp f_{0}\right)$ should be replaced by their finite-time equivalents $\delta_{T}\left(f \mp f_{0}\right)$ defined by

$\delta_{T}(f) \equiv \int_{-T / 2}^{T / 2} \mathrm{~d} t e^{-i 2 \pi f t}=\frac{\sin (\pi f T)}{\pi f} \equiv T \operatorname{sinc}(\pi f T)$,

where $T$ is the observation time for the pulsar. If one wants to also include the discreteness $\Delta t$ of the time-series data, then the Dirac delta functions should be replaced by Dirichlet sinc functions, $T \mathcal{D}_{N}\left[\left(f \mp f_{0}\right) \Delta t\right]$ [see (25)]. It turns out that the final (approximate) expressions that we obtain, cf. (45) and (49), are independent of which finitetime approximation we use.

\section{Averaging over inclination, polarization, and sky position}

Using the above expressions for $\tilde{h}_{+, \times}\left(f ; l, \phi_{0}\right)$ and (35) for $R^{+, \times}(f, \hat{k}, \psi)$, we can calculate the squared response $|\tilde{h}(f)|^{2}$ averaged over the inclination of the source (defined by the inclination and polarization angles $\imath$ and $\psi$ ), initial phase $\phi_{0}$, and sky direction $\hat{n} \equiv-\hat{k}$. This is relevant for the case where these quantities are not known a priori. Defining

$$
\begin{aligned}
|\tilde{h}(f ; \hat{k})|^{2} \equiv & \frac{1}{2 \pi} \int_{0}^{2 \pi} \mathrm{d} \phi_{0}\left(\frac{1}{4 \pi} \int_{0}^{2 \pi} \mathrm{d} \psi\right. \\
& \left.\times \int_{-1}^{1} \mathrm{~d}(\cos \iota)\left|\tilde{h}\left(f ; \hat{k}, \iota, \psi, \phi_{0}\right)\right|^{2}\right),
\end{aligned}
$$

it is fairly easy to show that 


$$
\frac{2|\tilde{h}(f ; \hat{k})|^{2}}{T} \simeq \frac{4}{5} \mathcal{R}(f, \hat{k}) S_{h}(f),
$$

where

$$
\begin{gathered}
\mathcal{R}(f, \hat{k}) \equiv \frac{1}{2}\left(\left|R^{+}(f, \hat{k}, 0)\right|^{2}+\left|R^{\times}(f, \hat{k}, 0)\right|^{2}\right), \\
S_{h}(f) \equiv \frac{1}{2} h_{0}^{2}\left[\delta\left(f-f_{0}\right)+\delta\left(f+f_{0}\right)\right] .
\end{gathered}
$$

The factor of $4 / 5$ in (45) comes from the average over inclination angles $(l, \psi) ; \mathcal{R}(f, \hat{k})$ encodes the timing residual response of a pulsar to a plane $\mathrm{GW}$ propagating in direction $\hat{k}$ averaged over the $(+, \times)$ polarizations and the polarization angle $\psi$; and $S_{h}(f)$ is the strain powerspectral density of a monochromatic GW having frequency $f_{0}$. The approximate equality in (45) is there because we made the approximation $\delta_{T}^{2}\left(f \mp f_{0}\right) \simeq T \delta\left(f \mp f_{0}\right)$ for the product of two finite-time Dirac delta functions. This allows us to write $S_{h}(f)$ in terms of ordinary Dirac delta functions, which are formally singular at $f= \pm f_{0}$. But this is not a problem, as $S_{h}(f)$ will only need to be evaluated under an integral sign for the expected signal-to-noise ratio calculations that we will perform in Sec. IVA. This approximation gives answers that are good to within $\lesssim 10 \%$ for noise power spectral densities that do not vary significantly over a frequency bandwidth $\Delta f \sim 1 / T$ in the neighborhood of $\pm f_{0}$.

If we also average over sky location, defining

$$
\begin{aligned}
|\tilde{h}(f)|^{2} \equiv & \frac{1}{4 \pi} \int \mathrm{d}^{2} \Omega_{\hat{k}}\left(\frac{1}{2 \pi} \int_{0}^{2 \pi} \mathrm{d} \phi_{0}\right. \\
& \left.\times\left(\frac{1}{4 \pi} \int_{0}^{2 \pi} \mathrm{d} \psi \int_{-1}^{1} \mathrm{~d}(\cos \iota)\left|\tilde{h}\left(f ; \hat{k}, \imath, \psi, \phi_{0}\right)\right|^{2}\right)\right),
\end{aligned}
$$

we find

$$
\frac{2|\tilde{h}(f)|^{2}}{T} \simeq \frac{4}{5} \mathcal{R}(f) S_{h}(f),
$$

where

$$
\begin{aligned}
\mathcal{R}(f) & \equiv \frac{1}{8 \pi} \int \mathrm{d}^{2} \Omega_{\hat{k}}\left(\left|R^{+}(f, \hat{k}, 0)\right|^{2}+\left|R^{\times}(f, \hat{k}, 0)\right|^{2}\right) \\
& =\frac{1}{12 \pi^{2} f^{2}} .
\end{aligned}
$$

Note that the expression for $\mathcal{R}(f)$ is independent of the direction $\hat{p}$ to the pulsar. The above expressions will be used later on when defining the detection sensitivity curves in Sec. IV.

\section{B. Response to a stochastic GW background}

For a stochastic GW background, the metric perturbations can be written as a superposition of plane GWs having different frequencies $f$, polarizations $\{+, \times\}$, and propagation directions $\hat{k}$,

$$
\begin{aligned}
h_{a b}(t, \vec{x})= & \int \mathrm{d}^{2} \Omega_{\hat{k}} \int_{-\infty}^{\infty} \mathrm{d} f\left[\tilde{h}_{+}(f, \hat{k}) e_{a b}^{+}(\hat{k})\right. \\
& \left.+\tilde{h}_{\times}(f, \hat{k}) e_{a b}^{\times}(\hat{k})\right] e^{i 2 \pi f(t-\hat{k} \cdot \vec{x} / c)},
\end{aligned}
$$

where $e_{a b}^{+, \times}(\hat{k}) \equiv \epsilon_{a b}^{+, \times}(\hat{k}, 0)$. This is basically (32) but allowing for contributions from different propagation direction $\hat{k}$. Since we will assume that the sources producing the GW background have no preferred polarization direction or symmetry axis, we have set $\psi=0$ and $l=0$ in the expansion for $h_{a b}(t, \vec{x})$. The timing residual response of a pulsar to the background is then

$$
h(t)=\int_{-\infty}^{\infty} \mathrm{d} f \tilde{h}(f) e^{i 2 \pi f t},
$$

where

$\tilde{h}(f)=\int \mathrm{d}^{2} \Omega_{\hat{k}}\left[R^{+}(f, \hat{k}, 0) \tilde{h}_{+}(f ; \hat{k})+R^{\times}(f, \hat{k}, 0) \tilde{h}_{\times}(f ; \hat{k})\right]$

with $R^{+, \times}(f, \hat{k}, 0)$ given by (35). As discussed there, we will ignore the contribution of the pulsar term to the response function, except when calculating the autocorrelated power, which will have contributions from both the Earth-Earth and pulsar-pulsar autocorrelation terms.

The Fourier components $\tilde{h}_{+, x}(f ; \hat{k})$ that enter the plane wave expansion of the metric perturbations are random fields. Their quadratic expectation values completely define the statistical properties of the background, under the assumption that it is Gaussian-distributed. For simplicity, we will assume that the GW background is stationary, unpolarized, and isotropic, ${ }^{4}$ for which $\left\langle\tilde{h}_{P}(f ; \hat{k})\right\rangle=0$ and

$$
\left\langle\tilde{h}_{P}(f ; \hat{k}) \tilde{h}_{P^{\prime}}^{*}\left(f^{\prime} ; \hat{k}^{\prime}\right)=\frac{1}{16 \pi} S_{h}(f) \delta\left(f-f^{\prime}\right) \delta_{P P^{\prime}} \delta^{2}\left(\hat{k}, \hat{k}^{\prime}\right),\right.
$$

where $P=\{+, \times\}$. Here $S_{h}(f)$ is the (one-sided) strain power spectral density of the background (units of strain $^{2} / \mathrm{Hz}$ ), which is related to the dimensionless energy-density spectrum $\Omega_{\mathrm{gw}}(f)$ via

\footnotetext{
${ }^{4}$ See e.g., [30] for a review of analyses that drop these assumptions.
} 


$$
S_{h}(f)=\frac{3 H_{0}^{2}}{2 \pi^{2}} \frac{\Omega_{\mathrm{gw}}(f)}{f^{3}} .
$$

It is also common to describe the background in terms of its dimensionless characteristic strain defined by

$$
h_{c}(f) \equiv \sqrt{f S_{h}(f)}=A_{\mathrm{gw}}\left(f / f_{\mathrm{yr}}\right)^{\alpha},
$$

where the second equality assumes a power-law form for the background. Note that for a background produced by the cosmological population of SMBH binaries, $\alpha=-2 / 3$.

\section{GW contribution to the noise covariance matrix}

Using the above expressions for the timing residual response of a pulsar to a GW background, we can calculate the GW contribution to the noise covariance matrix when cross-correlating timing residuals associated with two Earth-pulsar baselines $I$ and $J$. Denoting the GW contributions to the two sets of timing residuals as $h_{I}(t)$ and $h_{J}(t)$, respectively, one can show that the covariance matrix is block-diagonal with components,

$$
C_{h, I J} \equiv\left\langle h_{I} h_{J}^{T}\right\rangle-\left\langle h_{I}\right\rangle\left\langle h_{J}^{T}\right\rangle=\chi_{I J} C_{h},
$$

where

$\chi_{I J} \equiv \frac{1}{2}+\frac{3}{2}\left(\frac{1-\hat{p}_{I} \cdot \hat{p}_{J}}{2}\right)\left[\ln \left(\frac{1-\hat{p}_{I} \cdot \hat{p}_{J}}{2}\right)-\frac{1}{6}\right]+\frac{1}{2} \delta_{I J}$,

and

$$
\begin{aligned}
& C_{h, i j}=\int_{0}^{f_{\mathrm{Nyq}}} \mathrm{d} f \cos \left[2 \pi f\left(t_{i}-t_{i}\right)\right] P_{h}(f), \\
& P_{h}(f)=\mathcal{R}(f) S_{h}(f)=\frac{A_{\mathrm{gw}}^{2}}{12 \pi^{2}}\left(\frac{f}{f_{\mathrm{yr}}}\right)^{2 \alpha} f^{-3} .
\end{aligned}
$$

The full noise covariance matrix, which includes contributions intrinsic to the pulsar and to the measurement process, is also block-diagonal with components,

$$
C_{I J}=\delta_{I J} C_{n, I}+C_{h, I J}
$$

Here $C_{n, I}$ is given by (59), but with the pulsar noise power spectral density $P_{n_{I}}(f)$ replacing $P_{h}(f)$. This last equation assumes that the noise contributions associated with different pulsars are not correlated with one another.

The quantity $\chi_{I J} \equiv \chi\left(\zeta_{I J}\right)$ defined in (58) is the value of the Hellings and Downs [32] curve $\chi(\zeta)$ for a pair of pulsars separated by angle $\zeta_{I J}=\cos ^{-1}\left(\hat{p}_{I} \cdot \hat{p}_{J}\right)$ (see Fig. 8). It arises when cross-correlating the GW-induced timing residuals for an unpolarized, isotropic GW background.

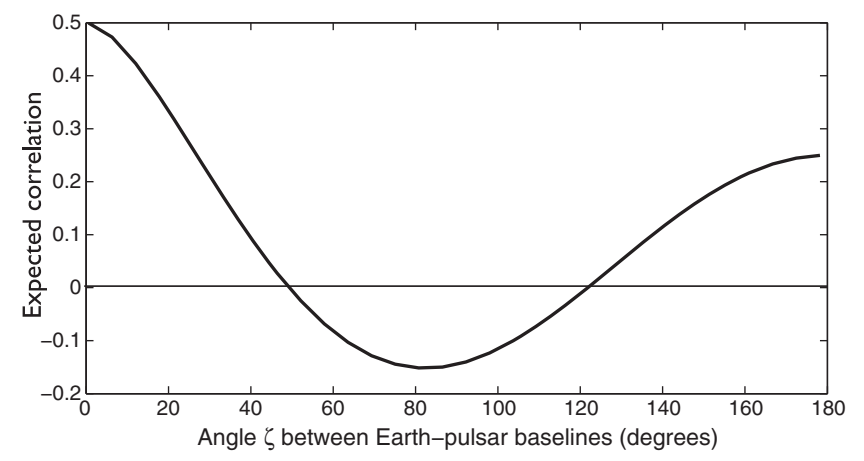

FIG. 8. Hellings and Downs curve $\chi(\zeta)$. Plotted is the expected correlation for the timing residuals induced in a pair of distinct Earth-pulsar baselines by an unpolarized, isotropic GW background.

Note that $\chi_{I J}$ has been normalized such that $\chi_{I I}=1$ (for a single pulsar).

\section{SENSITIVITY CURVES}

Ultimately, a detection sensitivity curve should tell us how likely it is to detect a particular type of GW signal. So it should depend not only on the properties of the noise in the detector, but also on the type of signal that one is searching for and the method that one uses to search for it. So here we extend the formalism of the previous two sections to define sensitivity curves for searches for a deterministic GW signal from a circular binary and an unpolarized, isotropic stochastic $\mathrm{GW}$ background. We begin by writing down expressions for the optimal detection statistics for these two different sources and their corresponding expected signal-to-noise ratios (SNRs) familiar from the literature in order to demonstrate how the inverse-noise-weighted transmission function, $\mathcal{N}^{-1}(f)$, appears in these expressions. We will see that from these expected SNRs, we can read off an effective strain-noise power spectral density, which has the interpretation of a detection sensitivity curve.

\section{A. Matched filtering for a deterministic GW signal}

For a deterministic GW signal, we can use the method of matched filtering to construct an optimal detection statistic. This method has been used extensively in the PTA literature, [28,33-35] and is also the basis for the approximate deterministic sensitivity curves in [26]. Letting $Q_{I}$ denote the filter function for pulsar $I$ (where $I=1,2, \ldots, N_{\mathrm{p}}$ ), we define

$$
\hat{\mathcal{S}} \equiv \sum_{I} Q_{I}^{T} r_{I}=\sum_{I} \sum_{\alpha} Q_{I \alpha} r_{I \alpha}
$$

where $r_{I} \equiv G_{I}^{T} \delta t_{I}$ are the TMM residuals for pulsar $I$. The filter function is determined by maximizing the expected signal-to-noise ratio, $\rho \equiv \mu / \sigma$, of $\hat{\mathcal{S}}$. The expectation value of $\hat{\mathcal{S}}$ is given by 


$$
\mu \equiv\langle\hat{\mathcal{S}}\rangle=\sum_{I} Q_{I}^{T} G_{I}^{T} h_{I}(\theta)
$$

and its variance is given by

$$
\sigma^{2} \equiv\left\langle\hat{\mathcal{S}}^{2}\right\rangle-\langle\hat{\mathcal{S}}\rangle^{2}=\sum_{I} Q_{I}^{T} \Sigma_{n, I} Q_{I}
$$

where $\Sigma_{n, I} \equiv G_{I}^{T} C_{n, I} G_{I}$ is the noise covariance matrix for $r_{I}$. This result for the variance assumes that the only GW contribution to the timing residuals is from a deterministic GW source, and not from a stochastic GW background. The presence of a stochastic background would contribute to both the diagonal and off diagonal block matrices [see (61)]. In what follows, we will assume that the off diagonal terms are small compared to the diagonal (autocorrelated) terms. But we will replace $\Sigma_{n, I}$ by $\Sigma_{I} \equiv G_{I}^{T} C_{I} G_{I}$, where $C_{I} \equiv C_{n, I}+C_{h}$, thereby allowing a stochastic background to contribute to the autocorrelated noise (sometimes called GW self-noise).

Using the above results for the mean and variance of $\hat{\mathcal{S}}$, the square of the expected signal-to-noise ratio is

$$
\rho^{2} \equiv \frac{\mu^{2}}{\sigma^{2}}=\frac{\sum_{I, J} Q_{I}^{T} G_{I}^{T} h_{I}(\theta) Q_{J}^{T} G_{J}^{T} h_{J}(\theta)}{\sum_{K} Q_{K}^{T} \Sigma_{K} Q_{K}}
$$

with the optimal filter given by

$$
\frac{\delta \rho^{2}}{\delta Q_{I}}=0 \Rightarrow Q_{I}=\Sigma_{I}^{-1} G_{I}^{T} h_{I}(\theta) .
$$

Note that $Q_{I}$ is a noise-weighted version of the TMM signal waveform, as expected for a matched-filter statistic. Using this expression, the expected signal-to-noise ratio becomes

$$
\begin{aligned}
\rho^{2}(\theta) & =\sum_{I} h_{I}(\theta)^{T} G_{I} \Sigma_{I}^{-1} G_{I}^{T} h_{I}(\theta) \\
& =\sum_{I} h_{I}(\theta)^{T} G_{I}\left(G_{I}^{T} C_{I} G_{I}\right)^{-1} G_{I}^{T} h_{I}(\theta) .
\end{aligned}
$$

By evaluating this last expression in the frequency domain, using (19) for $\mathcal{N}_{I}^{-1}\left(f, f^{\prime}\right)$ and restricting to the diagonal component $\mathcal{N}^{-1}(f)$ as discussed in Sec. II D,

$$
\rho^{2}(\theta) \simeq 4 \int_{0}^{f_{\mathrm{Nyq}}} \mathrm{d} f \sum_{I}\left|\tilde{h}_{I}(f ; \theta)\right|^{2} \mathcal{N}_{I}^{-1}(f) .
$$

Recall that $\theta$ denote the set of GW parameters. This expression is now written in a form where the formalism of Sec. II D is easily used. For the case of a circular binary discussed in Sec. III A $1, \theta=\left\{\hat{k}, \imath, \psi, \phi_{0}\right\}$.

\section{Detection sensitivity curve for sky and inclination-averaged sources}

To proceed further, we first consider the case of GWs from a single binary system averaged over the initial phase, inclination of the source, as well as its sky location. Using (49) for $\left|\tilde{h}_{I}(f)\right|^{2}$, we have

$$
\begin{aligned}
\left\langle\rho^{2}\right\rangle_{\text {inc,sky }} & \simeq 4 \int_{0}^{f_{\mathrm{Nyq}}} \mathrm{d} f \sum_{I} \frac{T_{I}}{2} \frac{4}{5} \mathcal{R}(f) S_{h}(f) \mathcal{N}_{I}^{-1}(f) \\
& =2 T_{\mathrm{obs}} \int_{0}^{f_{\mathrm{Nyq}}} \mathrm{d} f \frac{S_{h}(f)}{S_{\text {eff }}(f)}
\end{aligned}
$$

where

$$
\begin{gathered}
S_{\text {eff }}(f) \equiv\left(\frac{4}{5} \sum_{I} \frac{T_{I}}{T_{\text {obs }}} \frac{1}{S_{I}(f)}\right)^{-1}, \\
S_{I}(f) \equiv \frac{1}{\mathcal{N}_{I}^{-1}(f) \mathcal{R}(f)},
\end{gathered}
$$

and $T_{\mathrm{obs}}$ is a timescale characteristic of the PTA, here chosen as the time span of the full data set. Here, $S_{I}(f)$ is the strain-noise power spectral density for pulsar $I$, and $S_{\text {eff }}(f)$ is an effective strain-noise power spectral density for an array of pulsars. Given how $S_{\text {eff }}(f)$ appears in the expression for the expected signal-to-noise ratio, we will use it, or its dimensionless characteristic strain,

$$
h_{\mathrm{c}}(f) \equiv \sqrt{f S_{\mathrm{eff}}(f)},
$$

as a sensitivity curve for detecting a deterministic GW source averaged over its initial phase, inclination, and sky location. A plot of $S_{\text {eff }}(f)$ for the array of pulsars in the NANOGrav 11-year data [36] is shown in Fig. 9. Our calculation uses the noise parameters of the pulsars to build the covariance matrix used in calculating $\mathcal{N}^{-1}(f)$, as discussed in Sec. IV B. In addition, the sky positions, TOAs, and TOA errors are used. Note that for a monochromatic source, $S_{h}(f)$ has a very simple form given by (47), which implies

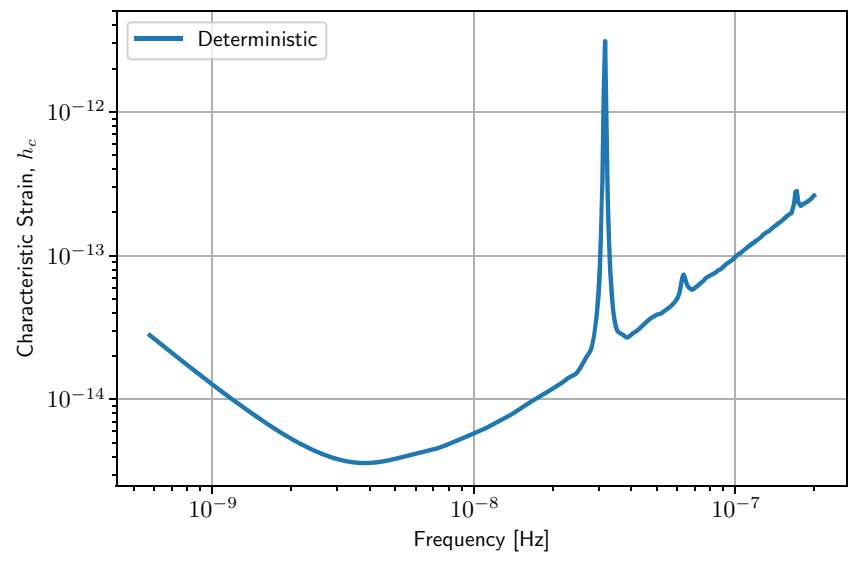

FIG. 9. Sensitivity curve for a single deterministic GW source averaged over its initial phase, inclination, and sky location. This plot was constructed using the NANOGrav 11-year data. 


$$
\bar{\rho} \equiv \sqrt{\left\langle\rho^{2}\right\rangle_{\text {inc,sky }}} \simeq h_{0} \sqrt{\frac{T_{\text {obs }}}{S_{\text {eff }}\left(f_{0}\right)}} .
$$

\section{SNR and characteristic amplitude sky maps for inclination-averaged sources}

If we average over initial phase and source inclination, but not over sky location, cf. (45) for $\left|\tilde{h}_{I}(f, \hat{k})\right|^{2}$, we obtain

$$
\begin{aligned}
\left\langle\rho^{2}(\hat{k})\right\rangle_{\mathrm{inc}} & \simeq 4 \int_{0}^{f_{\mathrm{Nyq}}} \mathrm{d} f \sum_{I} \frac{T_{I}}{2} \frac{4}{5} \mathcal{R}_{I}(f, \hat{k}) S_{h}(f) \mathcal{N}_{I}^{-1}(f) \\
& =2 T_{\mathrm{obs}} \int_{0}^{f_{\mathrm{Nyq}}} \mathrm{d} f \frac{S_{h}(f)}{S_{\mathrm{eff}}(f, \hat{k})}
\end{aligned}
$$

where

$$
\begin{gathered}
S_{\text {eff }}(f, \hat{k}) \equiv\left(\frac{4}{5} \sum_{I} \frac{T_{I}}{T_{\text {obs }}} \frac{1}{S_{I}(f, \hat{k})}\right)^{-1}, \\
S_{I}(f, \hat{k}) \equiv \frac{1}{\mathcal{N}_{I}^{-1}(f) \mathcal{R}_{I}(f, \hat{k})},
\end{gathered}
$$

with $\mathcal{R}_{I}(f, \hat{k})$ given by (46). These expressions are analogous to (70), but with added dependence on the propagation direction $\hat{k}$ of the GW. It turns out that we can factor out the $\hat{k}$ dependence on the right-hand side of the above expression for $S_{\text {eff }}(f, \hat{k})$ if we ignore the frequency-dependent part of the pulsar-term contribution to $\left|R_{I}^{P}(f, \hat{k}, 0)\right|^{2}$, as discussed in the context of (36). Making this approximation,

$$
\begin{aligned}
S_{\mathrm{eff}}(f, \hat{k}) \simeq & \left(\frac{12}{5} \sum_{I} \frac{T_{I}}{T_{\mathrm{obs}}} \frac{1}{S_{I}(f)}\right. \\
& \left.\times\left[\left(F_{I}^{+}(\hat{k})\right)^{2}+\left(F_{I}^{\times}(\hat{k})\right)^{2}\right]\right)^{-1},
\end{aligned}
$$

where $F_{I}^{+, \times}(\hat{k})$ are defined by

$$
F_{I}^{+, \times}(\hat{k}) \equiv \frac{1}{2} \frac{\hat{p}_{I}^{a} \hat{p}_{I}^{b}}{1+\hat{p}_{I} \cdot \hat{k}} e_{a b}^{+, \times}(\hat{k}) .
$$

As before, it is easy to do the integral over frequency for a monochromatic source, for which $S_{h}(f)$ is given by (47). The result is

$$
\rho(\hat{n}) \equiv \sqrt{\left\langle\rho^{2}(\hat{k})\right\rangle_{\mathrm{inc}}} \simeq h_{0} \sqrt{\frac{T_{\mathrm{obs}}}{S_{\mathrm{eff}}\left(f_{0}, \hat{k}\right)}},
$$

where the direction $\hat{n}$ of the source on the sky is opposite the direction of GW propagation, $\hat{n}=-\hat{k}$. A plot of $\rho(\hat{n})$

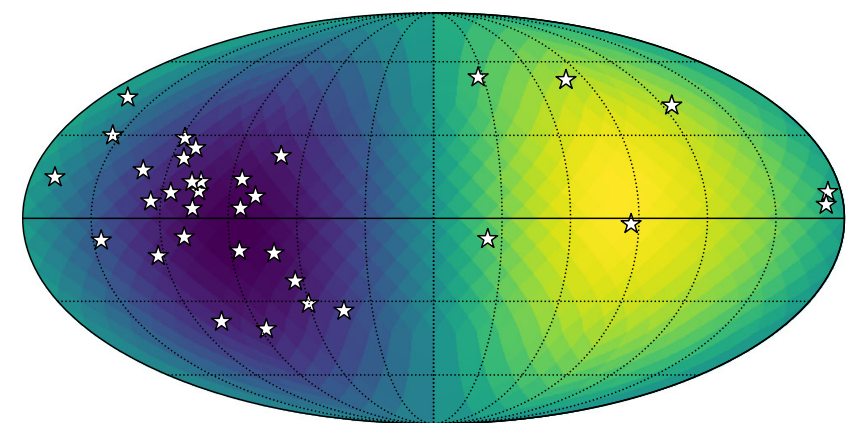

$$
\begin{array}{llllll}
1.0 & 1.5 & 2.0 & 2.5 & 3.0 & 3.5
\end{array}
$$

FIG. 10. Sky map of the expected matched-filter signal-to-noise ratio $\rho(\hat{n})$ for a monochromatic circular binary (GW frequency $f_{0}=8 \mathrm{nHz}$ ) consisting of a pair of $10^{9}$ solar-mass BHs at a luminosity distance of $100 \mathrm{Mpc}$. The stars show the locations of the NANOGrav pulsars. This plot was constructed using the NANOGrav 11-year data.

for a pair of $10^{9}$ solar-mass BHs at a luminosity distance of $100 \mathrm{Mpc}$, emitting monochromatic GWs at the frequency $f_{0}=8 \mathrm{nHz}$ is shown in Fig. 10 .

Finally, it is a simple matter to recast the form of the sky map so that we solve (79) for the strain amplitude $h_{0}$ of a monochromatic binary, cf. (41), that would produce a particular value of the signal-to-noise ratio $\rho$,

$$
h_{0}(\hat{n})=\rho \sqrt{\frac{S_{\mathrm{eff}}\left(f_{0}, \hat{k}\right)}{T_{\mathrm{obs}}}} .
$$

A sky map of $h_{0}(\hat{n})$ is shown in panel (a) of Fig. 11 for $\rho=2$ using the NANOGrav 11-year data. For comparison, panel (b) shows the actual 95\% confidence-level upper limit map taken from the NANOGrav 11-yr single-source paper [37]. Our sensitivity calculation gives $h_{0}=1.82 \times$ $10^{-15}$ at a right ascension and declination of $\left(17.6 \mathrm{~h}, 0^{\circ}\right)$ for the most sensitive sky location, while the full NANOGrav Bayesian analysis gives $h_{0}=1.82 \times 10^{-15}$ at a right ascension and declination of $\left(17.6 \mathrm{~h}, 9.6^{\circ}\right)$. This agreement is impressive in the sense that the full Bayesian analysis done by the NANOGrav collaboration took thousands of cpu-hours to complete, whereas the calculation using our formalism takes less than ten minutes on a laptop. It is unsurprising in the sense that, since PTAs have not made a detection of GWs, the statistics of PTAs are still dominated by the pulsar noise, observation cadence and PTA configuration, all characteristics used in the sensitivity curve calculation.

\section{B. Single-pulsar characteristic strain noise curves}

For an individual pulsar, we will use the characteristic strain, 

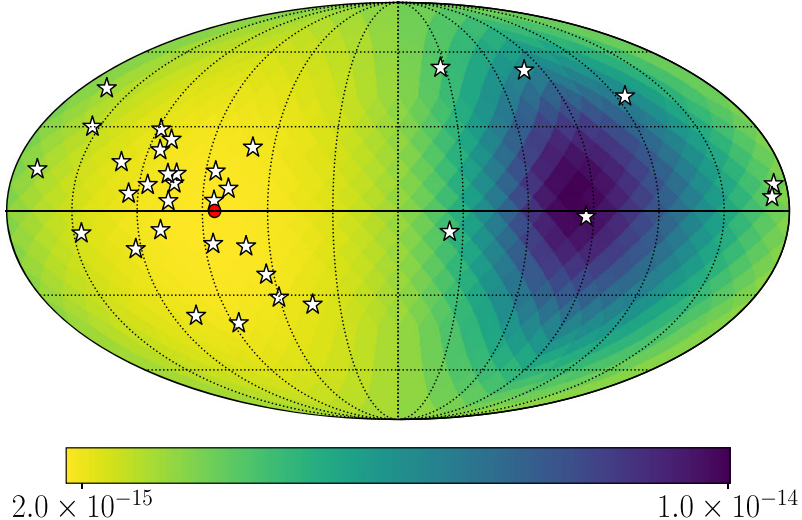

(a)
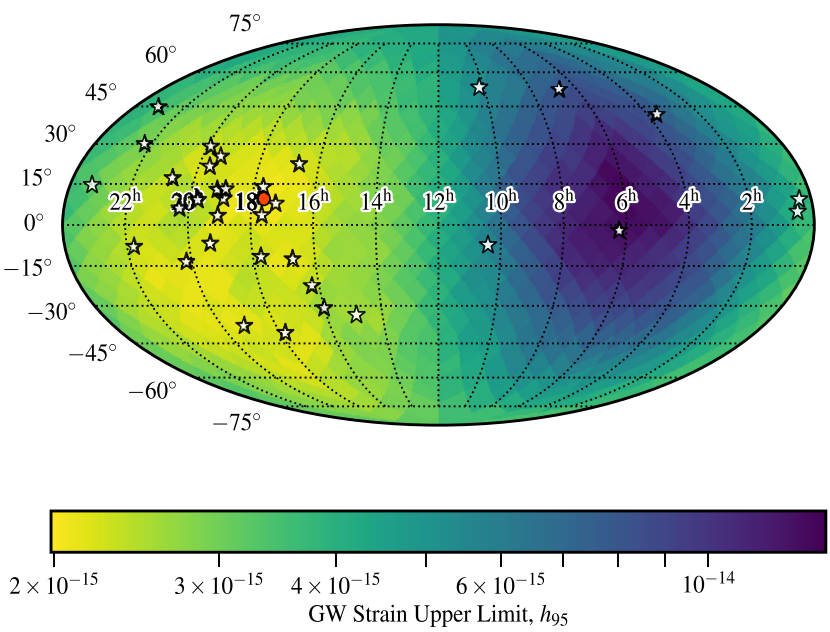

(b)

FIG. 11. Panel (a): Sensitivity sky map for the strain amplitude of a monochromatic continuous-wave source, calculated using the NANOGrav 11-yr data [38]. The stars show the locations of the NANOGrav pulsars and the red dot shows the most sensitive sky location. For this plot, we have taken $f_{0}=8 \mathrm{nHz}$ and $\rho=2$. Panel (b): For comparison, a 95\% confidence-level upper limit sky map taken from the NANOGrav 11-yr singlesource paper [37].

$$
h_{c, I}(f) \equiv \sqrt{f S_{I}(f)}, \quad S_{I}(f) \equiv \frac{1}{\mathcal{N}_{I}^{-1}(f) \mathcal{R}(f)},
$$

to characterize its polarization and sky-averaged sensitivity; see (71). Plots of single-pulsar characteristic strain-noise sensitivity curves for the simple quadratic spin-down model described in Sec. II A and for both white and red + white noise are shown in Fig. 12. More realistic single-pulsar strain-noise sensitivity curves can be constructed using a subset of the NANOGrav 11-year pulsars (Fig. 13) [36]. These pulsars have noise contributions specified by the parameters EQUAD, ECORR, and EFAC [36,38-40], which are denoted by $Q, J_{i j}$, and $F$ in the following expression for the noise covariance matrix:

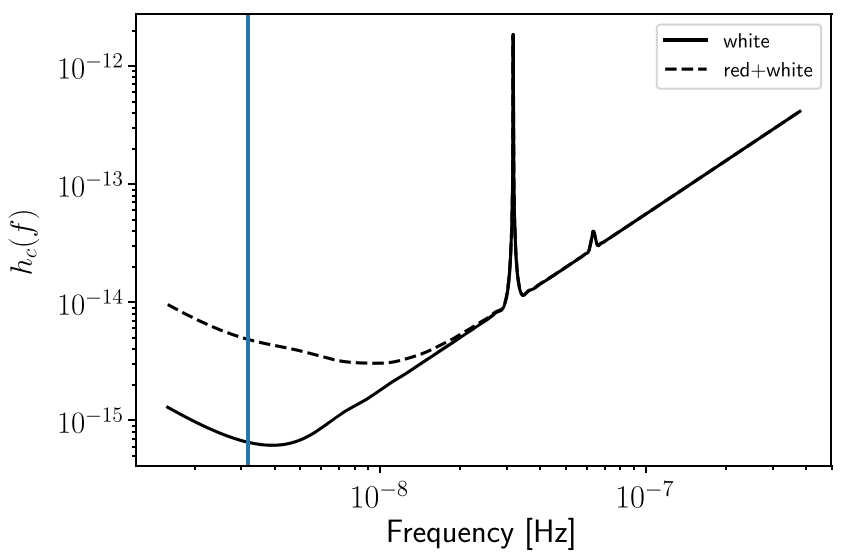

FIG. 12. Single-pulsar characteristic strain-noise sensitivity curves for the simple quadratic spin-down timing model fit described in the main text and for white noise (solid curve) and red + white noise (dashed curve). The vertical blue line corresponds to a frequency of $1 / T$.

$$
C_{n, i j}=F^{2}\left[\sigma_{i}^{2} \delta_{i j}\right]+Q^{2} \delta_{i j}+J_{i j}
$$

Here $\sigma_{i}^{2}$ are individual TOA errors, which are associated with the finite-signal-to-noise ratio determination of the pulse arrival times (obtained by correlating the observed pulses with a pulse template). EQUAD are white noise contributions to the covariance matrix that add in quadrature with the TOA errors. EFAC is an overall scale factor that can be used to adjust the overall uncertainty if necessary. ECORR are noise contributions that are correlated within an observing epoch, but not from epoch to epoch. Hence the ECORR contributions to the covariance matrix are block diagonal. Red noise, modeled as a power law, was added for those pulsars that show significant detections in the NANOGrav 11-yr data set [36]. In Fig. 13, $\mathrm{B} 1937+21$, J1713 + 0747 and J1909-3744 have injections of red noise. This can be distinguished by the "flatter" appearance of the sensitivity curves around the minimum, as compared to the other pulsars. For a detailed list of noise parameters, and to see which pulsars have significant detections of red noise, consult Table 2 in [38].

The NANOGrav 11-yr pulsars also have more complicated timing model fits than the simple quadratic spindown model described in Sec. II A. In Fig. 13, one can see that pulsar J1024-0719 is fit to a cubic spin-down model, leading to a steeper frequency-dependence $\left(\sim f^{-5 / 2}\right)$ at low frequencies. One also sees that $\mathrm{J} 1713+0747$ and $\mathrm{J} 1853+$ 1303 are in binary systems: there are additional spikes at the binary orbital frequency and twice the binary orbital frequency for $\mathrm{J} 1853+1303$. Finally, these pulsars have timing models that also include fits to a piecewise-constant, time-dependent dispersion measure fluctuation (DMX), which is associated with perturbations of the dispersion of the radio pulses as they propagate through the interstellar medium from the pulsar to a radio receiver on Earth. 

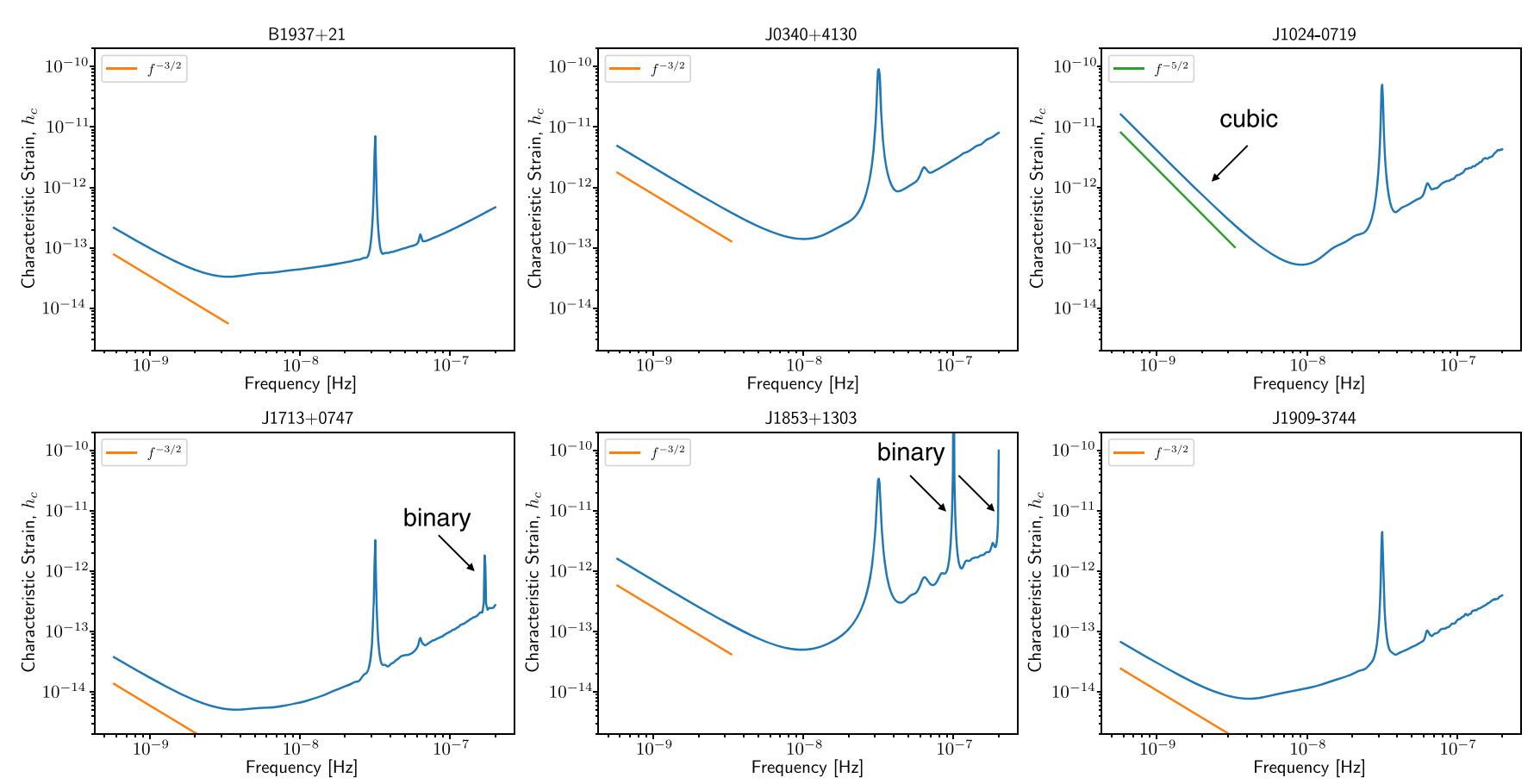

FIG. 13. Single-pulsar characteristic strain-noise sensitivity curves for a subset of NANOGrav $11-\mathrm{yr}$ pulsars. The $\sim f^{-5 / 2}$ behavior for PSR J1024-0719 is evidence of a fit to a cubic spin-down model for the pulsar spin frequency. The cubic term in the fit is needed due to an acceleration of the pulsar, evident in the TOAs from its unusually long binary period [36,41]. The additional spikes seen for $\mathrm{J} 1713+0747$ and $\mathrm{J} 1853+1303$ show that the pulsar is in a binary system; the second binary spike for $\mathrm{J} 1853+1303$ is the second harmonic of the binary orbital frequency.

[The lower-(radio)frequency components of a pulse are delayed more than the higher-frequency components.] Fitting to DMX in the timing model leads to broadband absorption of power relative to a timing model that does not fit for DMX. A piecewise-constant fit to the dispersion measure variations allows processes with all timescales

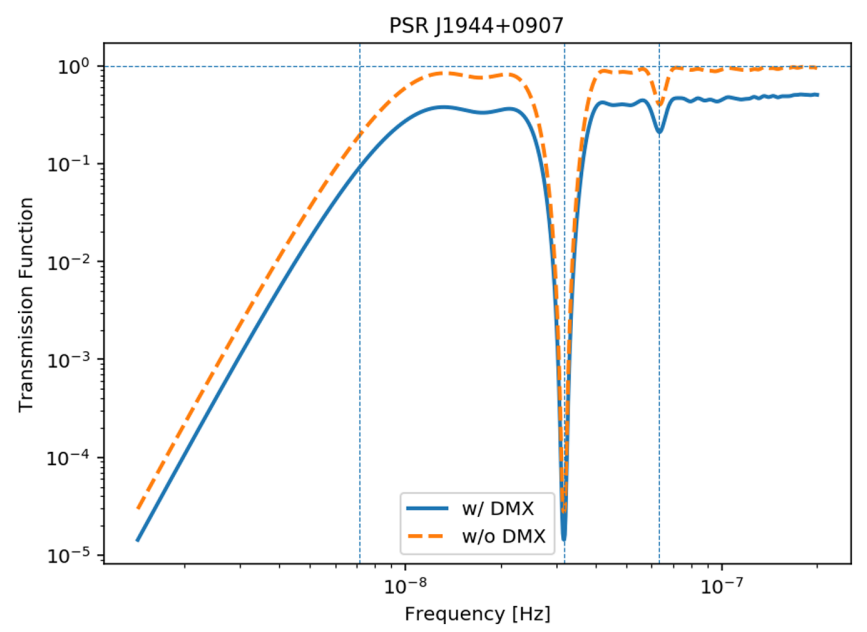

FIG. 14. Plots of transmission functions showing the effect of including time-dependent dispersion measure (DMX) into the timing model fit. Including DMX in the timing model leads to broadband absorption of power (solid blue curve) relative to that for a timing model without DMX. represented in the data to be removed: from the largetimescale variations, due to the slow movement of the interstellar medium, down to the short-timescale changes from the scintillation and scattering of the radio pulses. Figure 14 shows plots of the transmission function for NANOGrav pulsar J1944 + 0907, with and without DMX included in the timing model. Other models exist for dispersion measure variations and are treated exhaustively in [42]. The transmission function's dependence on these models depends greatly on the type of model being used. Alternatively, one can trade out the effect of dispersion measure variations on the transmission function by making the dispersion measure variation model part of the noise analysis, rather than part of the marginalized timing model, effectively replacing the power loss with uncertainty in the dispersion measure model parameters; see, e.g., [27,43].

\section{Optimal cross-correlation statistic for a stochastic GW background}

The derivation of the optimal cross-correlation statistic for a stochastic GW background is similar to that presented above for a single deterministic GW, expect that we work with data from pairs of pulsars. Starting with a single distinct pair, labeled by $I$ and $J$, we define

$$
\hat{\mathcal{S}}_{I J} \equiv r_{I}^{T} Q r_{J}
$$


where $r_{I}$ and $r_{J}$ are the TMM residuals for pulsars $I$ and $J$ (assuming that we have already fit for all deterministic GW sources), and $Q$ is an $m_{I} \times m_{J}$ matrix, where $m_{I} \equiv N_{I}-N_{\mathrm{par}, I}$, etc. As before, we determine the filter function $Q$ by maximizing the signal-to-noise ratio of $\hat{\mathcal{S}}_{I J}$. Similar derivations appear in the literature $[23,28,44-46]$. The final result for the optimal filter is

$$
Q \propto \Sigma_{I}^{-1} \Sigma_{I J} \Sigma_{J}^{-1}
$$

where

$$
\begin{aligned}
\Sigma_{I} & \equiv G_{I}^{T}\left(C_{n, I}+C_{h}\right) G_{I}, \\
\Sigma_{I J} & \equiv \chi_{I J} G_{I}^{T} C_{h} G_{J} .
\end{aligned}
$$

The expected squared signal-to-noise ratio for this optimal choice of $Q$ is then

$$
\rho_{I J}^{2}=\operatorname{Tr}\left[\Sigma_{J I} \Sigma_{I}^{-1} \Sigma_{I J} \Sigma_{J}^{-1}\right]
$$

The above calculation assumes that we are in the weaksignal limit where the cross-correlation terms are assumed to be negligible compared to autocorrelation terms (i.e., we assume that the GW signal power is much less than that for the intrinsic pulsar and measurement noise).

We can then combine the signal-to-noise ratios for each distinct pair in quadrature since, in the weak-signal limit, there is negligible correlation between these estimators,

$$
\rho^{2} \simeq \sum_{I} \sum_{J>I} \rho_{I J}^{2}
$$

As we saw for deterministic GWs, it is useful to write the above expression for the expected squared signal-to-noise ratio in the frequency domain. Proceeding as we did there, we find

$\rho^{2} \simeq \sum_{I} \sum_{J>I} 2 T_{I J} \chi_{I J}^{2} \int_{0}^{f_{\mathrm{Nyq}}} \mathrm{d} f S_{h}^{2}(f) \mathcal{R}^{2}(f) \mathcal{N}_{I}^{-1}(f) \mathcal{N}_{J}^{-1}(f)$,

where $P_{h}(f)=\mathcal{R}(f) S_{h}(f)$, and where $\mathcal{N}_{I}^{-1}(f)$ is defined by (20). This suggests defining the following effective strain-noise power spectral density for the whole PTA:

$$
S_{\mathrm{eff}}(f)=\left(\sum_{I} \sum_{J>I} \frac{T_{I J}}{T_{\mathrm{obs}}} \frac{\chi_{I J}^{2}}{S_{I}(f) S_{J}(f)}\right)^{-1 / 2},
$$

which includes contributions from the Hellings and Downs factors $\chi_{I J}$ and the individual pulsar strain-noise power spectral densities $S_{I}(f) \equiv 1 /\left(\mathcal{N}_{I}^{-1}(f) \mathcal{R}(f)\right)$. Note that $S_{\text {eff }}(f)$ has dimensions of $\operatorname{strain}^{2} / \mathrm{Hz}$, and that

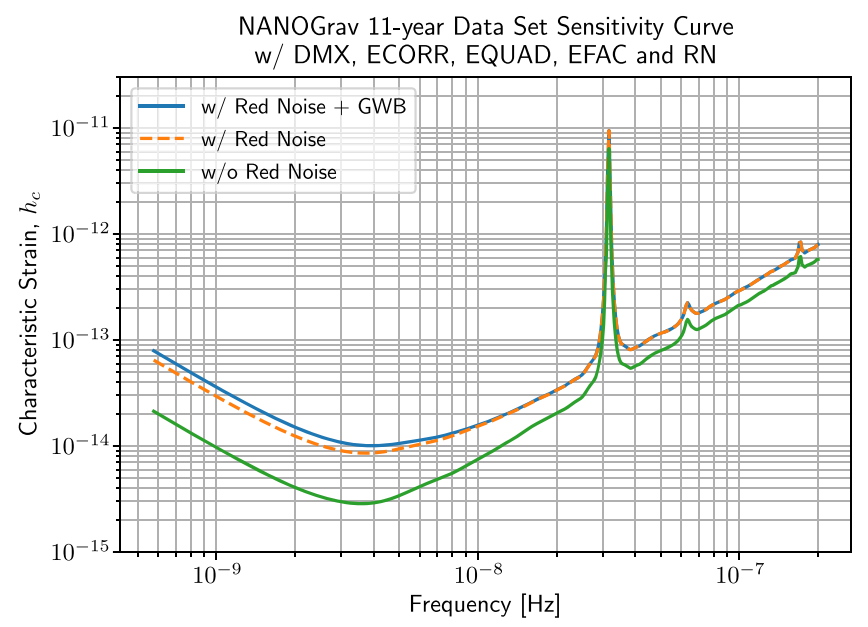

FIG. 15. Comparison of stochastic sensitivity curves (effective characteristic strain noise) for the NANOGrav 11-yr PTA. All the curves include realistic pulsar noise characteristics and individual timing model fits. The blue curve includes a contribution to the autopower spectra, produced by a GWB at the level of $A_{\mathrm{gwb}}=1 \times 10^{-16}$. The dashed-orange curve shows the sensitivity without including the GWB, and the green curve shows what happens if you also ignore the red noise contributions to the noise covariance matrices.

$$
\rho^{2} \simeq 2 T_{\text {obs }} \int_{0}^{f_{\mathrm{Nyq}}} \mathrm{d} f \frac{S_{h}^{2}(f)}{S_{\text {eff }}^{2}(f)}
$$

in terms of $S_{\text {eff }}(f)$.

A plot comparing dimensionless characteristic strain curves $h_{c}(f) \equiv \sqrt{f S_{\text {eff }}(f)}$ for stochastic GW backgrounds for the NANOGrav 11-yr pulsars is given in Fig. 15. The three curves show the effect of including a contribution from the GWB to the autopower spectra of all the pulsars (blue versus dashed-orange curves) and the false improvement in sensitivity that arises if one fails to include the rednoise component of the individual pulsar noise covariance matrices (green versus dashed-orange curves). Typical PTA sensitivity curves that one sees in the literature incorrectly ignore this red noise component, [13,26].

Since calculating sensitivity curves of this type is much more computationally efficient than doing real data analyses or analyzing simulated data with injected signals, another use of this formalism is making predictions about the sensitivity of PTAs into the future. Figure 16 shows a simulated PTA at various stages of maturity. All of the pulsars have a precision of $1 \mu \mathrm{s}$ and red noise corresponding to a GWB of $A_{\mathrm{GWB}}=6 \times 10^{-16}$. Half of the pulsars in each curve additionally have power-law red noise with $A_{\mathrm{RN}}=10^{-13}$ and $\gamma=2$. The array starts with 35 pulsars that range in time span from 3 yrs to 10 yrs. The next sensitivity curve shows the sensitivity 10 yrs later, with the addition of 15 pulsars, with a minimum of $3 \mathrm{yr}$ baseline. The most sensitive curve shows the PTA another $10 \mathrm{yrs}$ later with 75 pulsars, now spanning from 3 yrs to $30 \mathrm{yrs}$. 


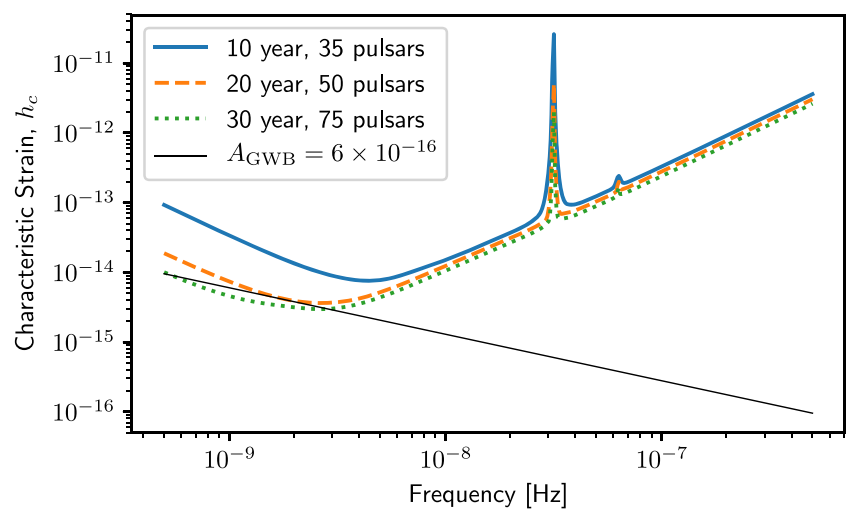

FIG. 16. A simulated pulsar timing array with sensitivity curves shown at 10, 20 and 30 years into its observing campaign. All pulsars have $1 \mu \mathrm{s}$ precision and a GWB of $A_{\mathrm{GWB}}=6 \times 10^{-16}$. Half of the pulsars, for each time span, additionally have powerlaw red noise with $A_{\mathrm{RN}}=10^{-13}$ and $\gamma=2$.

\section{Comparing stochastic and deterministic sensitivity curves}

Although one uses different statistics to search for deterministic and stochastic GW signals, it is interesting to compare the sensitivity curves for these two different cases. Figure 17 shows plots of the deterministic and stochastic sensitivity curves for the NANOGrav 11-yr pulsars (taken from Fig. 9 and Fig. 15, dashed-orange curve). Note that the sensitivity curve for a single deterministic source is lower than that for a stochastic background, since the Hellings and Downs factors $\chi_{I J}$ in (89) reduce the effective number of pulsar pairs that contribute to the stochastic analysis. To demonstrate this explicitly, compare Eqs. (70) and (89) for $S_{\text {eff }}(f)$ assuming that all the pulsars have the same noise characteristics and timing model fits [i.e., $S_{I}(f) \equiv S(f)$ for all $I$ ], and that all the pulsars are observed for the full observation time (i.e., $T_{I} \equiv T_{I J} \equiv T_{\text {obs }}$ ). Then,

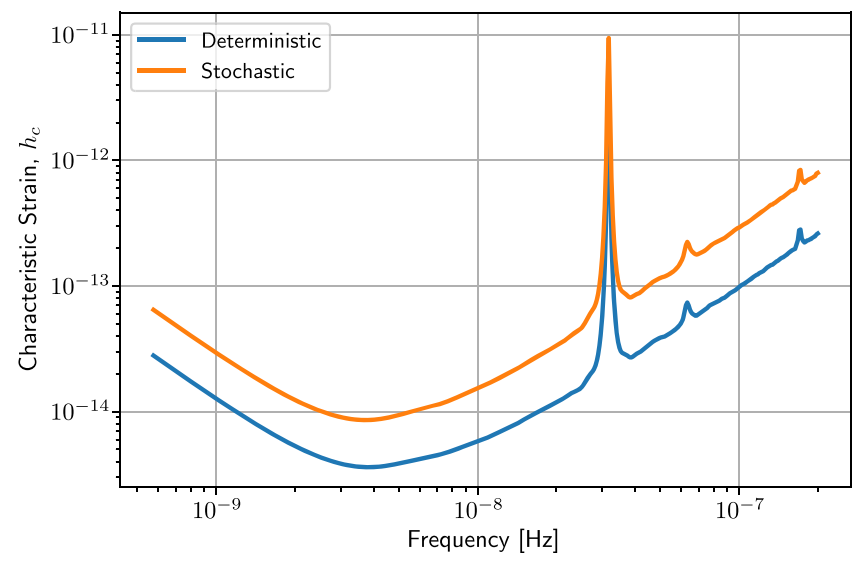

FIG. 17. Comparison of the sensitivity curves for the NANOGrav 11-yr pulsars to a single deterministic GW signal and a stochastic GW background; see also Figs. 9 and 15. The two curves differ by a factor of $\sim 2.6$.

$$
\begin{gathered}
S_{\mathrm{eff}}^{\mathrm{det}}(f)=\frac{5}{4 N_{\mathrm{p}}} S(f), \\
S_{\mathrm{eff}}^{\mathrm{stoch}}(f)=\left(\sum_{I} \sum_{J>I} \chi_{I J}^{2}\right)^{-1 / 2} S(f),
\end{gathered}
$$

where $N_{\mathrm{p}}$ is the number of pulsars. Since the maximum value of $\chi_{I J}$ for any pair of pulsars is $1 / 2$, we have

$$
\sum_{I} \sum_{J>I} \chi_{I J}^{2} \leq \frac{N_{\mathrm{p}}\left(N_{\mathrm{p}}-1\right)}{2} \frac{1}{4}
$$

which implies

$$
\left(\sum_{I} \sum_{J>I} \chi_{I J}^{2}\right)^{-1 / 2}>\frac{2 \sqrt{2}}{N_{\mathrm{p}}}
$$

Thus,

$$
S_{\text {eff }}^{\text {stoch }}(f)>\frac{2 \sqrt{2}}{N_{\mathrm{p}}} S(f) \Rightarrow S_{\text {eff }}^{\text {stoch }}(f)>S_{\text {eff }}^{\mathrm{det}}(f) .
$$

Although we have compared the full sensitivity curves $S_{\text {eff }}(f)$ for deterministic and stochastic GW sources, we note that the corresponding signal-to-noise ratio for a monochromatic deterministic source uses only the value of the sensitivity curve at a single frequency $f=f_{0}$ [see (73)]; while that for a stochastic source involves an integral of $S_{\text {eff }}(f)$ over all $f$ [see (90) and the discussion in Sec. IV C 3].

\section{Pairwise stochastic sensitivity curves}

As a by-product of the stochastic sensitivity curve analysis, we obtain pairwise stochastic sensitivity curves,

$$
h_{c, I J} \equiv \sqrt{f S_{I J}(f)}, \quad S_{I J}(f) \equiv \sqrt{\frac{T_{\mathrm{obs}}}{T_{I J}}} \frac{\sqrt{S_{I}(f) S_{J}(f)}}{\left|\chi_{I J}\right|},
$$

by simply restricting ourselves to a single term in the sum (89). Plots of such curves are useful as a diagnostic for comparing the contribution of different pulsar pairs to the stochastic optimal statistic signal-to-noise ratio. Figure 18 shows pairwise sensitivity curves for a subset of the NANOGrav 11-yr pulsars, comparing pairwise correlations of some of the most and least sensitive NANOGrav pulsars.

\section{Power-law integrated sensitivity curves}

For stochastic backgrounds that have a power-law spectrum, cf. (56), it is possible to construct a sensitivity curve that takes into account the improvement in sensitivity that comes from integrating over frequency [13]. Given a range of power-law indices, one determines the amplitude 


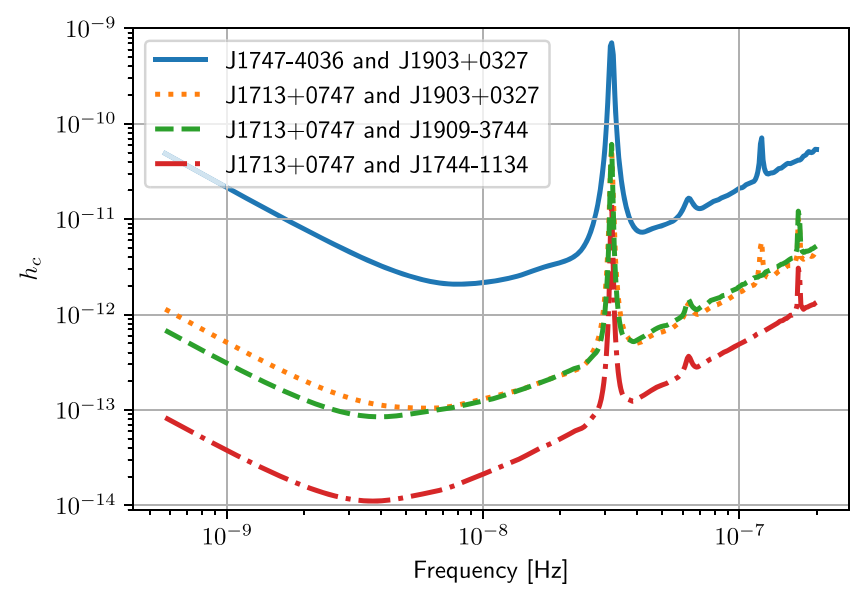

FIG. 18. Pairwise stochastic sensitivity curves (effective characteristic strain noise) for a subset of NANOGrav 11-yr pulsar pairs. Since pulsars J1747-4036 and J1903 + 0327 are two of the least-sensitive pulsars in the NANOGrav 11-yr data set, their pairwise sensitivity curve is worse (that is, higher) than the other pairs shown here. The sensitivity curve for J1713+0747 and $\mathrm{J} 1903+0327$ is significantly better, since $\mathrm{J} 1713+0747$ is the most sensitive pulsar in the data set; while that for $\mathrm{J} 1713+0747$ and J1744-1134 is the best, since both of these pulsars are individually very sensitive and their angular correlation is $\chi_{I J}=0.3304$. Pulsars J1713 +0747 and J1909-3744 are also both individually very sensitive, but since their angular correlation is only $\chi_{I J}=0.0058$, their pairwise sensitivity curve is an order of magnitude worse than that for $\mathrm{J} 1713+0747$ and J1744-1134.

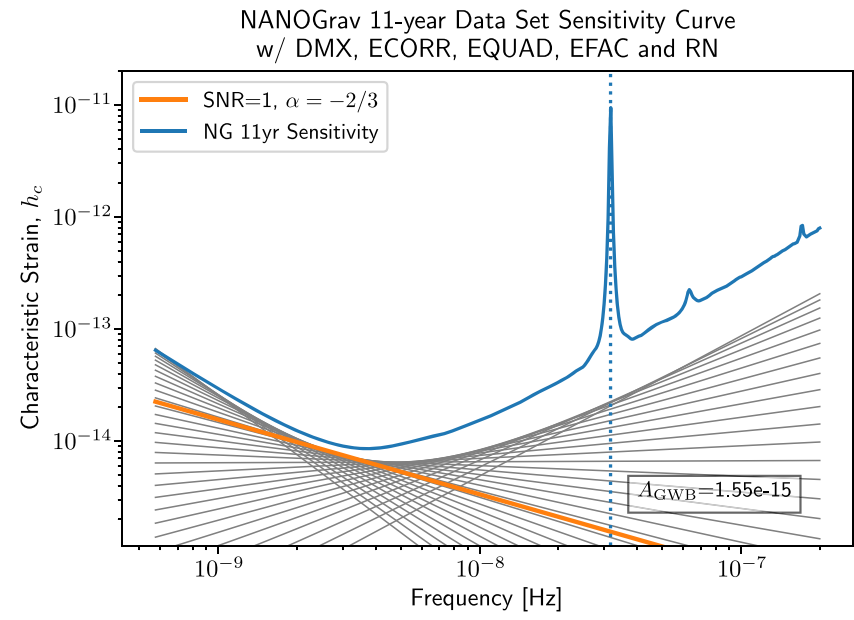

FIG. 19. Power-law-integrated sensitivity curve for the NANOGrav 11-yr data set. The blue line is the stochastic sensitivity curve for the NANOGrav 11-yr data set. Each of the straight grey lines represents a power-law GWB detectable with an optimalstatistic signal-to-noise ratio $\rho=1$ for the plotted spectral index. The envelope of these lines (i.e., the maximum value of all the power-law backgrounds at a given frequency) defines the powerlaw-integrated sensitivity curve for the PTA. The orange line is the power-law with spectral index of $\alpha=-2 / 3$, corresponding to a GWB background, at the amplitude needed for a detection with $\mathrm{SNR}=1$; in this case, $A_{\mathrm{GWB}}=1.55 \times 10^{-15}$. of each power-law background that yields a prescribed value of the optimal statistic signal-to-noise ratio $\rho$ (e.g., $\rho=1$ ). The envelope of these power-law backgrounds defines the power-law-integrated sensitivity curve for the PTA. Figure 19 shows the $\rho=1$ power-law integrated sensitivity curve for the NANOGrav 11-yr data set using the dashedorange characteristic strain-noise curve from Fig. 15. For the expected GWB spectral index of $\alpha=-2 / 3$ we calculate an amplitude of $A_{\mathrm{GWB}}=1.55 \times 10^{-15}$ needed to obtain $\mathrm{SNR}=1$. Compare this to the full NANOGrav 11-yr Bayesian analysis $95 \%$ credible upper limit value of $A_{\mathrm{GWB}}=$ $1.67 \times 10^{-15}$ and the frequentist optimal statistic results of $A_{\mathrm{GWB}}=9.8 \pm 4.7 \times 10^{-16}$ and $\mathrm{SNR}=0.8 \pm 0.7$. Our result is within $10 \%$ of the Bayesian result and reasonably close to the optimal statistic results, given that both the SNR and amplitude have error bars.

\section{DISCUSSION}

We have presented a method for constructing realistic detection sensitivity curves for pulsar timing arrays, valid for both deterministic and stochastic GW signals. We can include different noise characteristics and the effect of fitting to a timing model via an inverse-noise-weighted transmission function $\mathcal{N}_{I}^{-1}(f) \approx \mathcal{T}_{I}(f) / P(f)$. Singlepulsar sensitivity curves are then calculated from the strainnoise power spectral density $S_{I}(f) \equiv 1 /\left(\mathcal{N}_{I}^{-1}(f) \mathcal{R}(f)\right)$, where $\mathcal{R}(f)$ is the polarization and sky-averaged timing residual response of a pulsar to a passing GW. Detection sensitivity curves for multiple pulsars (i.e., a PTA) are similarly constructed from an effective strain-noise power spectral density $S_{\text {eff }}(f)$, which is a combination of singlepulsar strain-noise power spectral densities $S_{I}(f)$, cf. (70), (75), (89), appropriate for the GW source that one is interested in detecting.

The realistic sensitivity curves that we have calculated can be used to assess the detectability of different GW signals by existing or planned PTAs. This is in contrast to the usual approach in the literature where PTA sensitivity curves are computed using a highly simplified model. The computational cost of producing these sensitivity curves is minimal; they can be calculated much faster than analyzing simulated data containing injected signals. By properly incorporating realistic noise properties and the effect of timing model fits into the sensitivity curves, we can produce detectability estimates that agree quite well with the more computationally involved calculations.

\section{ACKNOWLEDGMENTS}

J. S. H. and J. D. R. acknowledge subawards from the University of Wisconsin-Milwaukee for the NSF NANOGrav Physics Frontier Center (NSF Grant No. PFC-1430284). J. D. R. also acknowledges support from start-up funds from Texas Tech University. T. L. S. acknowledges support from NASA Grant No. 80NSSC18K0728 and the Hungerford 
Fund at Swarthmore College. Finally, we thank Robert Caldwell, Rutger van Haasteren, and Xavi Siemens for useful discussions and Justin Ellis for sharing some preliminary code.

\section{APPENDIX: CASTING THE BLANDFORD ET $A L$. ANALYSIS [16] IN MORE MODERN NOTATION}

When using pulsar timing data to search for GWs, one needs to take into account the effects of fitting to a deterministic timing model when doing any type of additional signal analysis. Following [16], we define the residuals $R(t)$ as the difference between the observed arrival times of the pulses and the expected arrival times as determined by our best guesses to the parameters. These residuals are fit to an expression linear in the corrections to the unknown parameters, $\alpha_{a}{ }^{5}$ (Noise terms are added later in their analysis.) We start in the notation of [16] and then translate to expressions in terms of modern PTA GW analyses,

$$
R(t)=\sum_{a=1}^{N_{\mathrm{par}}} \alpha_{a} \psi_{a}(t) .
$$

We will define $R_{i} \equiv R\left(t_{i}\right)$, which is a vector of length $N$, and $\psi_{i a} \equiv \psi_{a}\left(t_{i}\right)$, which is a two-dimensional matrix with dimensions $N \times N_{\mathrm{par}}$. (Note we have reversed the order of the indices on $\psi_{i a}$ from that in [16], to be consistent with later work.) In more modern PTA data analysis papers, like [22] or [23,47], this matrix is referred to as the design matrix of the timing model (our $M_{i a}$.) The above expression for the residuals can be transformed into an orthonormal basis,

$$
R_{i}=\sum_{a=1}^{N_{\mathrm{par}}} \alpha_{a}^{\prime} \psi_{i a}^{\prime}, \quad \psi_{i a}^{\prime} \equiv \sum_{b=1}^{N_{\mathrm{par}}} \psi_{i b} L_{b a},
$$

where

$$
\sum_{i=1}^{N} \psi_{a i}^{\prime T} \psi_{i b}^{\prime}=\delta_{a b} .
$$

Using these definitions we calculate a relation that will be useful in the next section. To simplify the notation a bit we will use the Einstein convention of summing over repeated indices without including summation symbols, using matrix transposes where necessary. Thus, for example, the orthonormality conditions can be written as

$$
\delta_{a b}=\psi_{a i}^{\prime T} \psi_{i b}^{\prime}=L_{a c}^{T} \psi_{c i}^{T} \psi_{i d} L_{d b} .
$$

Since a change of basis change is invertible, we can act with the inverse transformation matrices,

\footnotetext{
${ }^{5}$ In our notation, $R(t)$ is $\delta t_{i}$ and $\alpha_{a}$ is $\delta \xi_{a}$.
}

$$
\begin{aligned}
L_{e a}^{-T} \delta_{a b} L_{b f}^{-1} & =L_{e a}^{-T} L_{a c}^{T} \psi_{c i}^{T} \psi_{i d} L_{d b} L_{b f}^{-1}, \\
L_{e a}^{-T} L_{a f}^{-1} & =\psi_{e i}^{T} \psi_{i f},
\end{aligned}
$$

where $L^{-T}$ denotes the inverse of the transpose matrix $L^{T}$, which is the same as the transpose of the inverse matrix $L^{-1}$. Finally, using the well-known identity for the inverse of a product of two matrices,

$$
\left(L_{e a}^{-T} L_{a f}^{-1}\right)^{-1}=\left(\psi_{e i}^{T} \psi_{i f}\right)^{-1} \Rightarrow L_{f a} L_{a e}^{T}=\left(\psi_{e i}^{T} \psi_{i f}\right)^{-1} .
$$

\section{Least-squares regression}

One finds the best fit to a timing model by minimizing a $\chi^{2}$ function, which we will define below. In [16] an ordinary least squares (OLS) minimization is used. In subsequent PTA papers a weighted-least-squares (WLS) regression is used, where each residual is weighted by the inverse of the TOA error, $W_{i} \equiv 1 / \sigma_{i}$. In the most modern work a generalized least squares (GLS) regression is used where the noise covariance matrix, $N_{i j}$, is used, encoding covariances between all residuals,

$$
\chi^{2} \equiv\left(R_{i}-\alpha_{a}^{\prime} \psi_{a i}^{\prime T}\right) N_{i j}^{-1}\left(R_{j}-\psi_{j b}^{\prime} \alpha_{b}^{\prime}\right) .
$$

Here we solve the GLS minimization problem, restricting to simpler scenarios if needed-i.e., $N_{i j}^{-1}=\sigma_{i}^{-2} \delta_{i j}$ for the case of WLS, and $N_{i j}^{-1}=\delta_{i j}$ for OLS (as noise is not taken into account during the OLS fit). We minimize the expression for $\chi^{2}$ above by finding the $\operatorname{root}(\mathrm{s})$ of the derivative with respect to the parameters,

$$
\begin{aligned}
0 & =\frac{\partial \chi^{2}}{\partial \alpha_{a}^{\prime}} \\
& =-\psi_{a i}^{\prime T} N_{i j}^{-1} R_{j}+\psi_{a i}^{\prime T} N_{i j}^{-1} \psi_{j b}^{\prime} \alpha_{b}^{\prime}+(\text { transpose }) .
\end{aligned}
$$

Solving for $\alpha_{b}^{\prime}$ gives

$$
\alpha_{b}^{\prime}=\left(\psi_{a i}^{\prime T} N_{i j}^{-1} \psi_{j b}^{\prime}\right)^{-1} \psi_{a k}^{\prime T} N_{k l}^{-1} R_{l} .
$$

In [16], they consider OLS fitting. There the noise is taken into account after the fit, but its existence is implicit throughout. For instance the difference between the lhs and rhs side of their Eq. (2.9) would be zero if there was no noise. Setting $N_{i j}=\delta_{i j}$ gives

$$
\alpha_{b}^{\prime}=\left(\psi_{a i}^{\prime T} \psi_{i b}^{\prime}\right)^{-1} \psi_{a j}^{\prime T} R_{j}=\delta_{a b}^{-1} \psi_{a j}^{\prime T} R_{j}=\psi_{b i}^{\prime T} R_{i} .
$$

This is the result that [16] reports for the best fit. For WLS fitting, we have

$$
\alpha_{b}^{\prime}=\left(\psi_{a i}^{\prime T} W_{i j}^{2} \psi_{j b}^{\prime}\right)^{-1} \psi_{a k}^{\prime T} W_{k l}^{2} R_{l},
$$

where $W_{i j}^{2} \equiv \sigma_{i}^{-2} \delta_{i j}$. 


\section{Transmission function for ordinary least-squares regression}

The transmission function is defined by [16] as the transfer function relating the power in the prefit residuals $R_{i}$ to that in the postfit residuals,

$$
R_{i}^{\text {post }} \equiv R_{i}-\psi_{i a}^{\prime} \alpha_{a}^{\prime},
$$

where $\alpha_{a}^{\prime}$ are the best-fit values to the parameter deviations, determined by the $\chi^{2}$ minimization procedure discussed above. For the case of OLS fitting, which [16] consider, $\alpha_{a}^{\prime}$ is given by (A10), implying

$$
R_{i}^{\text {post }} \equiv R_{i}-\psi_{i a}^{\prime} \psi_{a j}^{\prime T} R_{j}=\left(\delta_{i j}-\psi_{i a}^{\prime} \psi_{a j}^{\prime T}\right) R_{j} .
$$

The variance in the postfit residual is then

$$
\begin{aligned}
\sigma_{\text {post }}^{2} & \equiv \frac{1}{N}\left\langle R^{\text {post } T} R^{\text {post }}\right\rangle \\
& =\frac{1}{N}\left\langle R_{j} R_{k}\right\rangle\left(\delta_{j i}-\psi_{j a}^{\prime} \psi_{a i}^{\prime T}\right)\left(\delta_{i k}-\psi_{i b}^{\prime} \psi_{b k}^{\prime T}\right) \\
& =\frac{1}{N}\left\langle R_{j} R_{k}\right\rangle\left(\delta_{j k}-\psi_{j a}^{\prime} \psi_{a k}^{\prime T}-\psi_{j b}^{\prime} \psi_{b k}^{\prime T}+\psi_{j a}^{\prime} \psi_{a i}^{\prime T} \psi_{i b}^{\prime} \psi_{b k}^{\prime T}\right) \\
& =\frac{1}{N}\left\langle R_{j} R_{k}\right\rangle\left(\delta_{j k}-\psi_{j a}^{\prime} \psi_{a k}^{\prime T}\right),
\end{aligned}
$$

where we used orthogonality of the $\psi_{j a}^{\prime}$ to get the last line. Since the covariance matrix $\left\langle R_{i} R_{j}\right\rangle$ is related to its power spectral density $P(f)$ via

$$
\left\langle R_{i} R_{j}\right\rangle=\int_{0}^{\infty} \mathrm{d} f P(f) e^{i 2 \pi f\left(t_{i}-t_{j}\right)},
$$

it follows that

$$
\sigma_{\text {post }}^{2}=\int_{0}^{\infty} \mathrm{d} f \mathcal{T}(f) P(f),
$$

where

$$
\begin{aligned}
\mathcal{T}(f) & \equiv 1-\frac{1}{N} \psi_{i a}^{\prime} \psi_{a j}^{\prime T} e^{i 2 \pi f\left(t_{i}-t_{j}\right)} \\
& =1-\frac{1}{N} \tilde{\psi}_{a}^{\prime}(f)\left(\tilde{\psi}_{a}^{\prime}(f)\right)^{\dagger}
\end{aligned}
$$

with $\tilde{\psi}_{a}^{\prime}$ the Fourier transforms of the basis functions,

$$
\tilde{\psi}_{a}^{\prime}(f)=\psi_{i a}^{\prime} e^{i 2 \pi f t_{i}} .
$$

Making this substitution and transforming $\psi_{i a}^{\prime}$ back to the original basis, we find

$$
\begin{aligned}
\mathcal{T}(f) & =1-\frac{1}{N} \psi_{i b} L_{b a} L_{a c}^{T} \psi_{c j}^{T} e^{i 2 \pi f\left(t_{i}-t_{j}\right)} \\
& =1-\frac{1}{N} \psi_{i b}\left(\psi_{c k}^{T} \psi_{k b}\right)^{-1} \psi_{c j}^{T} e^{i 2 \pi f\left(t_{i}-t_{j}\right)} \\
& =\frac{1}{N}\left(\delta_{i j}-\psi_{i b}\left(\psi_{c k}^{T} \psi_{k b}\right)^{-1} \psi_{c j}^{T}\right) e^{i 2 \pi f\left(t_{i}-t_{j}\right)},
\end{aligned}
$$

which is an expression for transmission function in terms of the original design matrix $\psi_{i a}$.

\section{Transmission function for generalized least-squares regression}

For the case of GLS fitting, the best-fit values for the timing parameter deviations are given by (A9), for which the postfit residuals are given by

$$
\begin{aligned}
R_{i}^{\text {post }} & =R_{i}-\psi_{i a}^{\prime}\left(\psi_{b j}^{\prime T} N_{j k}^{-1} \psi_{k a}^{\prime}\right)^{-1} \psi_{b m}^{\prime T} N_{m j}^{-1} R_{j} \\
& =\left(\delta_{i j}-\psi_{i a}^{\prime}\left(\psi_{b k}^{\prime T} N_{k l}^{-1} \psi_{l a}^{\prime}\right)^{-1} \psi_{b m}^{\prime T} N_{m j}^{-1}\right) R_{j} .
\end{aligned}
$$

We can write this in terms of the original basis as

$$
\begin{aligned}
R_{i}^{\text {post }} & =\left(\delta_{i j}-\psi_{i c} L_{c a}\left(L_{b e}^{T} \psi_{e k}^{T} N_{k l}^{-1} \psi_{l d} L_{d a}\right)^{-1} L_{b f}^{T} \psi_{f m}^{T} N_{m j}^{-1}\right) R_{j} \\
& =\left(\delta_{i j}-\psi_{i c} L_{c a} L_{a d}^{-1}\left(\psi_{e k}^{T} N_{k l}^{-1} \psi_{l d}\right)^{-1} L_{e b}^{-T} L_{b f}^{T} \psi_{f m}^{T} N_{m j}^{-1}\right) R_{j} \\
& =\left(\delta_{i j}-\psi_{i d}\left(\psi_{e k}^{T} N_{k l}^{-1} \psi_{l d}\right)^{-1} \psi_{e m}^{T} N_{m j}^{-1}\right) R_{j},
\end{aligned}
$$

which has exactly the same form as (A20) with $\psi_{i a}^{\prime}$ replaced by $\psi_{i a}$. The variance of the postfit residuals is thus

$$
\begin{aligned}
\sigma_{\text {post }}^{2} & \equiv \frac{1}{N}\left\langle R^{\text {post } T} R^{\text {post }}\right\rangle \\
& =\frac{1}{N}\left(\delta_{i j}-\psi_{i a}\left(\psi_{b l}^{T} N_{l m}^{-1} \psi_{m a}\right)^{-1} \psi_{b n}^{T} N_{n j}^{-1}\right)\left\langle R_{j} R_{k}\right\rangle\left(\delta_{k i}-N_{k q}^{-1} \psi_{q c}\left(\psi_{d r}^{T} N_{r s}^{-1} \psi_{s c}\right)^{-1} \psi_{d i}^{T}\right) .
\end{aligned}
$$

Since $\left\langle R_{i} R_{j}\right\rangle \equiv N_{j k}$ for GLS fitting, we get 


$$
\begin{aligned}
\sigma_{\mathrm{post}}^{2}= & \frac{1}{N}\left(N_{i k}-\psi_{i a}\left(\psi_{b l}^{T} N_{l m}^{-1} \psi_{m a}\right)^{-1} \psi_{b k}^{T}\right)\left(\delta_{k i}-N_{k q}^{-1} \psi_{q c}\left(\psi_{d r}^{T} N_{r s}^{-1} \psi_{s c}\right)^{-1} \psi_{d i}^{T}\right) \\
= & \frac{1}{N}\left(N_{i k} \delta_{k i}-\psi_{i a}\left(\psi_{b l}^{T} N_{l m}^{-1} \psi_{m a}\right)^{-1} \psi_{b i}^{T}-\psi_{i c}\left(\psi_{d r}^{T} N_{r s}^{-1} \psi_{s c}\right)^{-1} \psi_{d i}^{T}\right. \\
& \left.+\psi_{i a}\left(\psi_{b l}^{T} N_{l m}^{-1} \psi_{m a}\right)^{-1} \psi_{b k}^{T} N_{k q}^{-1} \psi_{q c}\left(\psi_{d r}^{T} N_{r s}^{-1} \psi_{s c}\right)^{-1} \psi_{d i}^{T}\right) \\
= & \frac{1}{N}\left(N_{i k} \delta_{k i}-\psi_{i a}\left(\psi_{b l}^{T} N_{l m}^{-1} \psi_{m a}\right)^{-1} \psi_{b i}^{T}\right) \\
= & \frac{1}{N}\left(\delta_{i j}-\psi_{i a}\left(\psi_{b l}^{T} N_{l m}^{-1} \psi_{m a}\right)^{-1} \psi_{b k}^{T} N_{k j}^{-1}\right) N_{i j},
\end{aligned}
$$

where we used the symmetry of $N_{i j}$ throughout. Finally, using (A15) for $N_{i j}$, we recover (A16) with

$$
\mathcal{T}(f)=\mathcal{T}_{R}(f) \equiv \frac{1}{N}\left(\delta_{i j}-\psi_{i a}\left(\psi_{b l}^{T} N_{l m}^{-1} \psi_{m a}\right)^{-1} \psi_{b k}^{T} N_{k j}^{-1}\right) e^{i 2 \pi f\left(t_{i}-t_{j}\right)} .
$$

We thus obtain the same $R$-matrix-dependent transmission function $\mathcal{T}_{R}(f)$ found in (15), with the $R$-matrix given by the expression in parentheses, $R_{i j} \equiv \delta_{i j}-\psi_{i a}\left(\psi_{b l}^{T} N_{l m}^{-1} \psi_{m a}\right)^{-1} \psi_{b k}^{T} N_{k j}^{-1}$.

[1] X. Siemens, J. Ellis, F. Jenet, and J.D. Romano, The stochastic background: Scaling laws and time to detection for pulsar timing arrays, Classical Quantum Gravity 30, 224015 (2013).

[2] P. A. Rosado, A. Sesana, and J. Gair, Expected properties of the first gravitational wave signal detected with pulsar timing arrays, Mon. Not. R. Astron. Sci. 451, 2417 (2015).

[3] S. R. Taylor, M. Vallisneri, J. A. Ellis, C. M. F. Mingarelli, T. J. W. Lazio, and R. van Haasteren, Are we there yet? Time to detection of Nanohertz gravitational waves based on Pulsar-timing array limits, Astrpohys. J. Lett. 819, L6 (2016).

[4] L. Z. Kelley, L. Blecha, L. Hernquist, A. Sesana, and S. R. Taylor, The gravitational wave background from massive black hole binaries in Illustris: Spectral features and time to detection with pulsar timing arrays, Mon. Not. R. Astron. Sci. 471, 4508 (2017).

[5] M. V. Sazhin, Opportunities for detecting ultralong gravitational waves, Soviet Astron. 22, 36 (1978).

[6] S. Detweiler, Pulsar timing measurements and the search for gravitational waves, Astrophys. J. 234, 1100 (1979).

[7] R. S. Foster and D. C. Backer, Constructing a pulsar timing array, Astrophys. J. 361, 300 (1990).

[8] B. P. Abbott et al. (LIGO Scientific and Virgo Collaborations), Observation of Gravitational Waves from a Binary Black Hole Merger, Phys. Rev. Lett. 116, 061102 (2016).

[9] B. P. Abbott et al. (LIGO Scientific and Virgo Collaboration), GWTC-1: A Gravitational-Wave Transient Catalog of Compact Binary Mergers Observed by LIGO and Virgo during the First and Second Observing Runs, Phys. Rev. X 9, 031040 (2019).

[10] B. P. Abbott et al. (LIGO Scientific and Virgo Collaborations), GW170817: Observation of Gravitational Waves from a Binary Neutron Star Inspiral, Phys. Rev. Lett. 119, 161101 (2017).
[11] C. J. Moore, R. H. Cole, and C. P. L. Berry, Gravitationalwave sensitivity curves, Classical Quantum Gravity 32, 015014 (2015).

[12] T. Robson, N. J. Cornish, and C. Liug, The construction and use of LISA sensitivity curves, Classical Quantum Gravity 36, 105011 (2019).

[13] E. Thrane and J. D. Romano, Sensitivity curves for searches for gravitational-wave backgrounds, Phys. Rev. D 88, 124032 (2013).

[14] G. Hobbs, Pulsars as gravitational wave detectors, Astrophys. Space Sci. Proc. 21, 229 (2011).

[15] J. M. Cordes, Pulsar timing. II. Analysis of random walk timing noise: Application to the Crab pulsar, Astrophys. J. 237, 216 (1980).

[16] R. Blandford, R. Narayan, and R. W. Romani, Arrival-time analysis for a millisecond pulsar, J. Astrophys. Astron. 5, 369 (1984).

[17] C. Cutler, S. Burke-Spolaor, M. Vallisneri, J. Lazio, and W. Majid, The gravitational-wave discovery space of Pulsar timing arrays, Phys. Rev. D 89, 042003 (2014).

[18] D. R. Madison, S. Chatterjee, and J. M. Cordes, The benefits of VLBI astrometry to Pulsar timing array searches for gravitational radiation, Astrophys. J. 777, 104 (2013).

[19] P. B. Demorest, Measuring the gravitational wave background using precision pulsar timing, Ph.D. thesis, University of California, 2007.

[20] R. van Haasteren, Y. Levin, P. McDonald, and T. Lu, On measuring the gravitational-wave background using Pulsar timing arrays, Mon. Not. R. Astron. Soc. 395, 1005 (2009).

[21] W. Coles, G. Hobbs, D. J. Champion, R. N. Manchester, and J.P.W. Verbiest, Pulsar timing analysis in the presence of correlated noise, Mon. Not. R. Astron. Sci. 418, 561 (2011).

[22] R. van Haasteren and Y. Levin, Understanding and analysing time-correlated stochastic signals in pulsar timing, Mon. Not. R. Astron. Soc. 428, 1147 (2013). 
[23] S. J. Chamberlin, J. D. E. Creighton, X. Siemens, P. Demorest, J. Ellis, L. R. Price, and J. D. Romano, Timedomain Implementation of the optimal cross-correlation statistic for stochastic gravitational-wave background searches in Pulsar timing data, Phys. Rev. D 91, 044048 (2015).

[24] K. J. Lee, C. G. Bassa, G. H. Janssen, R. Karuppusamy, M. Kramer, R. Smits, and B. W. Stappers, The optimal schedule for pulsar timing array observations, Mon. Not. R. Astron. Sci. 423, 2642 (2012).

[25] K. J. Lee, Prospects of gravitational wave detection using pulsar timing array for Chinese future telescopes, in Frontiers in Radio Astronomy and FAST Early Sciences Symposium 2015, Astronomical Society of the Pacific Conference Series, Vol. 502, edited by L. Qain and D. Li (Astronomical Society of the Pacific, Guiyang, China, 2016), p. 19.

[26] C. J. Moore, S. R. Taylor, and J. R. Gair, Estimating the sensitivity of pulsar timing arrays, Classical Quantum Gravity 32, 055004 (2015).

[27] R. N. Caballero, K. J. Lee, L. Lentati, G. Desvignes, D. J. Champion, J. P. W. Verbiest, G. H. Janssen, B. W. Stappers, M. Kramer, and P. Lazarus, The noise properties of 42 millisecond pulsars from the European Pulsar timing array and their impact on gravitational-wave searches, Mon. Not. R. Astron. Sci. 457, 4421 (2016).

[28] J. A. Ellis, X. Siemens, and J. D. E. Creighton, Optimal strategies for continuous gravitational wave detection in pulsar timing arrays, Astrophys. J. 756, 175 (2012).

[29] J. H. Taylor and J. M. Weisberg, A new test of general relativity: Gravitational radiation and the binary pulsar PS R 1913 + 16, Astrophys. J. 253, 908 (1982).

[30] J. D. Romano and N. J. Cornish, Detection methods for stochastic gravitational-wave backgrounds: A unified treatment, Living Rev. Relativity 20, 2 (2017).

[31] S. L. Detweiler, Pulsar timing measurements and the search for gravitational waves, Astrophys. J. 234, 1100 (1979).

[32] R. W. Hellings and G. S. Downs, Upper limits on the isotropic gravitational radiation background from pulsar timing analysis, Astrophys. J. 265, L39 (1983).

[33] S. Babak et al., European Pulsar timing array limits on continuous gravitational waves from individual supermassive black hole binaries, Mon. Not. R. Astron. Sci. 455, 1665 (2016).

[34] S. Babak and A. Sesana, Resolving multiple supermassive black hole binaries with pulsar timing arrays, Phys. Rev. D 85, 044034 (2012).

[35] D. R. B. Yardley, G. B. Hobbs, F. A. Jenet, J. P. W. Verbiest, Z. L. Wen, R. N. Manchester, W. A. Coles, W. van Straten,
M. Bailes, N. D. R. Bhat, S. Burke-Spolaor, D. J. Champion, A. W. Hotan, and J. M. Sarkissian, The sensitivity of the parkes Pulsar timing array to individual sources of gravitational waves, Mon. Not. R. Astron. Soc. 407, 669 (2010).

[36] Z. Arzoumanian et al. (NANOGrav Collaboration), The NANOGrav 11-year data set: High-precision timing of 45 millisecond Pulsars, Astrophys. J. Suppl. Ser. 235, 37 (2018).

[37] K. Aggarwal et al., The NANOGrav 11-year data set: Limits on Gravitational Waves from Individual Supermassive Black Hole Binaries, Astrophys. J. 880, 116 (2019).

[38] Z. Arzoumanian et al. (NANOGRAV Collaboration), The NANOGrav 11-year data set: Pulsar-timing constraints on the stochastic gravitational-wave background, Astrophys. J. 859, 47 (2018).

[39] M. T. Lam et al., The NANOGrav nine-year data set: Excess noise in millisecond Pulsar arrival times, Astrophys. J. 834, 35 (2017).

[40] Z. Arzoumanian et al. (NANOGrav Collaboration), Gravitational waves from individual supermassive black hole binaries in circular orbits: Limits from the north American Nanohertz observatory for gravitational waves, Astrophys. J. 794, 141 (2014).

[41] D. L. Kaplan et al., PSR J1024-0719: A millisecond Pulsar in an unusual long-period orbit, Astrophys. J. 826, 86 (2016).

[42] M. L. Jones et al., The NANOGrav nine-year data set: Measurement and analysis of variations in dispersion measures, Astrophys. J. 841, 125 (2017).

[43] L. Lentati et al., From spin noise to systematics: Stochastic processes in the first International Pulsar timing array data release, Mon. Not. R. Astron. Soc. 458, 2161 (2016).

[44] B. Allen and J. D. Romano, Detecting a stochastic background of gravitational radiation: Signal processing strategies and sensitivities, Phys. Rev. D 59, 102001 (1999).

[45] M. Anholm, S. Ballmer, J. D. E. Creighton, L. R. Price, and X. Siemens, Optimal strategies for gravitational wave stochastic background searches in pulsar timing data, Phys. Rev. D 79, 084030 (2009).

[46] S. J. Vigeland, K. Islo, J. A. Ellis, and S. R. Taylor, Noisemarginalized optimal statistic: A robust hybrid frequentistBayesian statistic for the stochastic gravitational-wave background in pulsar timing arrays, Phys. Rev. D 98, 044003 (2018)..

[47] J. A. Ellis, X. Siemens, and R. van Haasteren, An efficient approximation to the likelihood for gravitational wave stochastic background detection using Pulsar timing data, Astrophys. J. 769, 63 (2013). 